\title{
Tuberous sclerosis complex inactivation disrupts melanogenesis via mTORC1 activation
}

\author{
Juxiang Cao, ${ }^{1}$ Magdalena E. Tyburczy, ${ }^{1}$ Joel Moss, ${ }^{2}$ Thomas N. Darling, ${ }^{3}$ Hans R. Widlund, ${ }^{4}$ and David J. Kwiatkowski \\ 'Division of Pulmonary and Critical Care Medicine and Genetics, Department of Medicine, Brigham and Women's Hospital, Harvard Medical School, Boston, Massachusetts, USA. ²Cardiovascular and \\ Pulmonary Branch, National Heart, Lung, and Blood Institute (NHLBI), NIH, Bethesda, Maryland, USA. ${ }^{3}$ Department of Dermatology, Uniformed Services University of the Health Sciences, Maryland, USA. \\ ${ }^{4}$ Department of Dermatology, Brigham and Women's Hospital, Harvard Medical School, Boston, Massachusetts, USA.
}

\begin{abstract}
Tuberous sclerosis complex (TSC) is an autosomal dominant tumor-suppressor gene syndrome caused by inactivating mutations in either TSC1 or TSC2, and the TSC protein complex is an essential regulator of mTOR complex 1 (mTORC1). Patients with TSC develop hypomelanotic macules (white spots), but the molecular mechanisms underlying their formation are not fully characterized. Using human primary melanocytes and a highly pigmented melanoma cell line, we demonstrate that reduced expression of either TSC1 or TSC2 causes reduced pigmentation through mTORC1 activation, which results in hyperactivation of glycogen synthase kinase $3 \beta$ (CSK3 $\beta$ ), followed by phosphorylation of and loss of $\beta$-catenin from the nucleus, thereby reducing expression of microphthalmia-associated transcription factor (MITF), and subsequent reductions in tyrosinase and other genes required for melanogenesis. Genetic suppression or pharmacological inhibition of this signaling cascade at multiple levels restored pigmentation. Importantly, primary melanocytes isolated from hypomelanotic macules from 6 patients with TSC all exhibited reduced TSC2 protein expression, and 1 culture showed biallelic mutation in TSC2, one of which was germline and the second acquired in the melanocytes of the hypomelanotic macule. These findings indicate that the TSC/mTORC1/AKT/CSK3 $\beta / \beta$-catenin/MITF axis plays a central role in regulating melanogenesis. Interventions that enhance or diminish mTORC1 activity or other nodes in this pathway in melanocytes could potentially modulate pigment production.
\end{abstract}

\section{Introduction}

Tuberous sclerosis complex (TSC) is an autosomal dominant disorder characterized by the presence of hamartomas (distinctive tumors) in multiple organ systems including the brain, skin, eyes, heart, lungs, and kidneys, as well as by refractory epilepsy and a variety of neuropsychiatric manifestations $(1,2)$. TSC is caused by inactivating mutations in either TSC1 or TSC2 (3). TSC1 and TSC2, together with TBC1D7, form the TSC protein complex that functions as a GAP toward the GTPase Ras homolog enriched in brain (RHEB) (4). RHEB is a critical upstream regulator of mTOR complex 1 (mTORC1), which coordinately regulates multiple cellular pathways that control cell size and anabolic metabolism as a prerequisite for cell division (4-11). In cells lacking either TSC1 or TSC2, RHEB-GTP levels are high, leading to the constitutive activation of mTORC1 $(9,12)$. mTORC1 activation leads to a complex set of feedback or counterregulatory effects that inhibit PI3K/AKT signaling, which is demonstrated through reduced levels of phosphorylated AKT (p-AKT) (Ser473) and AKT kinase activity. These counterregulatory effects include phosphorylation and degradation of insulin receptor substrate 1 (IRS-1) by S6K1 (13-15); reduced expression of IRS-2 $(13,14)$; reduced expression of PDGFR (16); reduced activity of mTORC2, which phosphorylates AKT at Ser473 (17); and phosphorylation and inactivation of GRB10 by mTORC1 $(18,19)$.

Conflict of interest: The authors have declared that no conflict of interest exists. Submitted: August 14, 2015; Accepted: October 20, 2016.

Reference information: / Clin Invest. 2017;127(1):349-364. doi:10.1172/JCI84262.
Several types of skin lesions are common in TSC: hypopigmented macules (also called ash-leaf or white spots), facial angiofibroma, cephalic plaque, shagreen patches, and ungual fibromas $(2,20)$. We have recently shown that facial angiofibroma and other TSC cutaneous tumors are due to 2-hit mutational inactivation of both alleles of either TSC1 or TSC2 and that lesions on the face often sustain UV-induced CC>TT mutations in the second allele (21). Hypopigmented macules typically appear similar to an ash leaf in configuration, with relative hypopigmentation in comparison with surrounding skin, and are most commonly seen on the limbs and trunk $(22,23)$. Since these macules can be detected at birth, they were proposed as one of the earliest diagnostic markers of TSC 50 years ago (24). Ultrastructural studies have shown that melanocyte content in TSC hypomelanotic macules is normal, while intracellular pigment granules are reduced in size and number $(22,25)$. Despite these morphologic and histologic studies, however, the mechanism of TSC-associated hypopigmentation has remained unknown.

Melanin is produced by skin melanocytes and other anatomically restricted pigment cells in the eye and brain (26-31). In the pigment biosynthesis pathway, tyrosinase (encoded by TYR) catalyzes the initial and rate-limiting step in melanin production by converting L-tyrosine to L-3,4-dihydroxyphenylalanine (L-DOPA) $(32,33)$. The melanocyte-specific isoform of the microphthalmia-associated transcription factor M-MITM controls the transcription of $T Y R$ and other genes required for melanogenesis and melanosome formation (e.g., premelanosome protein $[P M E L]$, dopachrome tautomerase $[D C T]$, MelanA [MLANA]) 
through binding to conserved E-boxes [CAC(G/A)TG] or M-boxes [TCAC(G/A)TG] in their promoter regions. Mutations in MITF cause the autosomal dominant disorder Waardenburg syndrome type 2A (WS2A), in which aberrant pigmentation and other features are seen (34-36). Melanocyte lineage expression of MITF is conferred by the context-dependent activity of several transcription factors: paired box 3 (PAX3), cAMP-responsive element-binding protein (CREB), and $\beta$-catenin, in concert with $\mathrm{T}$ cell transcription factor/lymphoid enhancer-binding factor 1 (TCF/LEF1) (37, 38). WNT/ $\beta$-catenin signaling is required for the developmental pathway that leads to melanocyte derivation from the neural crest (39-41). Wnt ligand leads to phosphorylation and inhibition of glycogen synthase kinase $3 \beta$ (GSK3 $\beta$ ), disassembly of the destruction complex, an increase in $\beta$-catenin levels, and $\beta$-catenin translocation to the nucleus, where it binds to the TCF/LEF transcription factors to enhance the transcription of MITF and other targets $(42-46)$. AKT signaling also negatively regulates both GSK3 $\beta$ and $\operatorname{GSK} 3 \alpha(47,48)$. Insulin and other growth factors lead to activation of AKT, which phosphorylates GSK3 at Ser9 (GSK3 $\beta$ ) or Ser21 $(\mathrm{GSK} 3 \alpha)$, thereby inhibiting GSK kinase activity.

Connecting the earlier understanding of the migration of melanocyte precursors during development (49) with previous studies on the role of mTOR in the regulation of pigment (43, 50 ), we hypothesized that TSC hypopigmented macules occursbecause of a second-hit mutation and loss of TSC1/TSC2 occurring at the melanoblast stage of development, leading to clonal skin patches in which melanocytes have mTORC1 activation and reduced pigmentation. We show here that, in both melanocytes and melanoma cells, reduced expression of either TSC1 or TSC2 leads to inhibition of AKT, followed by enhanced, activated GSK3 $\beta$, which in turn leads to phosphorylation of $\beta$-catenin and a marked reduction in nuclear $\beta$-catenin levels, followed by reduced LEF1 transcriptional activity, reduced MITF transcription and expression, and, ultimately, markedly reduced melanogenesis. Importantly, we found that melanocyte cultures from hypomelanotic macules from several TSC patients had reduced TSC2 and activated $\mathrm{mTORC} 1$ - with biallelic mutation in TSC 2 in 1 patient - and were deficient in melanin production, which was consistent with our hypothesis.

\section{Results}

The TSC protein complex controls melanogenesis. To examine the role of the TSC protein complex in melanogenesis regulation, we studied immortalized human primary melanocytes (iHPMs) (51) and the relatively highly pigmented human melanoma cell line SK-MEL-30. Suppression of either TSC1 or TSC2 expression, using lentiviral shRNA, led to a marked reduction in visible pigmentation in iHPMs (Figure 1, B and F), with a reduction in melanin content (Figure 1, C and G) and TYR activity (Figure 1, D and H). Similar effects were seen in the SK-MEL-30 melanoma cell line (Supplemental Figure 1, B-D and F-H; supplemental material available online with this article; doi:10.1172/JCI84262DS1. See complete unedited blots in the supplemental material). The pigmentation reduction was proportional to the degree of TSC1 or TSC2 suppression seen across the 3 different shRNA constructs used for each gene (Figure 1, A-H and Supplemental Figure 1, A-H). As expected, the knockdown cells showed elevated levels of p-S6 (Ser235/236); p-S6 (Ser240/244); p-70S6K1 (Thr229); p-70S6K1 (Thr389); and p-4E-BP1 (Thr37/46), as well as reduced p-AKT (Thr308) and p-AKT (Ser473), all of which were consistent with mTORC1 activation and feedback inhibition of AKT signaling due to reduced expression of the TSC protein complex (Figure 1, A and E, and Supplemental Figure 1, A and E). TSC2 expression was reduced in the cell lines when TSC1 was reduced, as has been seen previously (10).

Because the transcription factor MITF controls expression of TYR and other enzymes in pigment biosynthesis, including PMEL, DCT, and TYRP1 (52), we asked whether suppression of TSC1/TSC2 affected its expression. MITF-M and its downstream target genes PGC-1 $\alpha$, TYR, TYRP1, PMEL, and DCT (53) showed reduced expression at the protein (Figure 1, A and E, and Supplemental Figure 1, A and E) and mRNA levels (Figure 1M and Supplemental Figure 1M; note that mRNA experiments were performed only for the shRNAs with the strongest effects: shTSC1-3 and shTSC2-2) in both iHPMs and SK-MEL-30 cells.

We sought to confirm that the loss of pigmentation was due to the loss of TSC1-TSC2 protein complex GAP activity toward RHEB. We expressed WT TSC2 or a TSC2 mutant (P419S) derived from a TSC patient with defective GAP activity in the TSC2-knockdown cell lines. WT, but not mutant TSC2 reduced mTORC1 activity, restored the expression of MITF, PGC- $1 \alpha$, and TYR, and restored TYR activity, melanin production, and pigmentation in iHPMs and SK-MEL-30 cells (Figure 1, I-L and Supplemental Figure $1, \mathrm{I}-\mathrm{L}$, respectively).

To confirm that the reduced pigmentation was due to effects on MITF expression in the TSC1/TSC2-knockdown cells, we expressed MITF-M in the cells expressing the most efficient TSC1 and TSC2 shRNAs. Ectopic MITF expression at a level similar to that in control cells restored both pigmentation and TYR protein expression to levels seen in the control cells (Figure 1, O and P, and Supplemental Figure 1, O and P).

TSC protein complex controls melanogenesis through inhibition of $m$ TORC1 activity. To confirm that the loss of pigmentation seen in these cell lines with shRNA against TSC1 or TSC2 was due to enhanced mTORC1 activity, we treated the cells with the mTORC1 allosteric inhibitor rapamycin. Rapamycin treatment (20 nM) rapidly blocked mTORC1 activity in the knockdown cells (data not shown), as seen with other cell types. After 4 days of rapamycin treatment, MITF expression was restored, as was that of its target genes PGC-1 $\alpha$ and TYR in both TSC1- and TSC2-knockdown cells (Figure 2, A and E, and Supplemental Figure 2, A and E). Visual assessment of pigmentation, TYR activity, and melanin content of the rapamycin-treated cells confirmed that all were restored to baseline levels (Figure 2, B-D and F-H; and Supplemental Figure 2, B-D, and $\mathrm{F}-\mathrm{H})$. We also noted that rapamycin-treated control cells increased their pigmentation to a minor extent (Figure 2, C and G, and Supplemental Figure 2, C and G). Previous studies have shown that rapamycin treatment elevates the levels of MITF and its target genes TYR, TRYP1, and PMEL in mouse melanoma cells $(54,55)$, human primary melanocytes, and human melanoma cells (43), though to a modest extent (<2-fold). Combined with our results, this suggested that it was specifically mTORC1 activity due to TSC1/TSC2 loss that was leading to reduced MITF expression and thus reduced pigmentation. To confirm our results, we also 


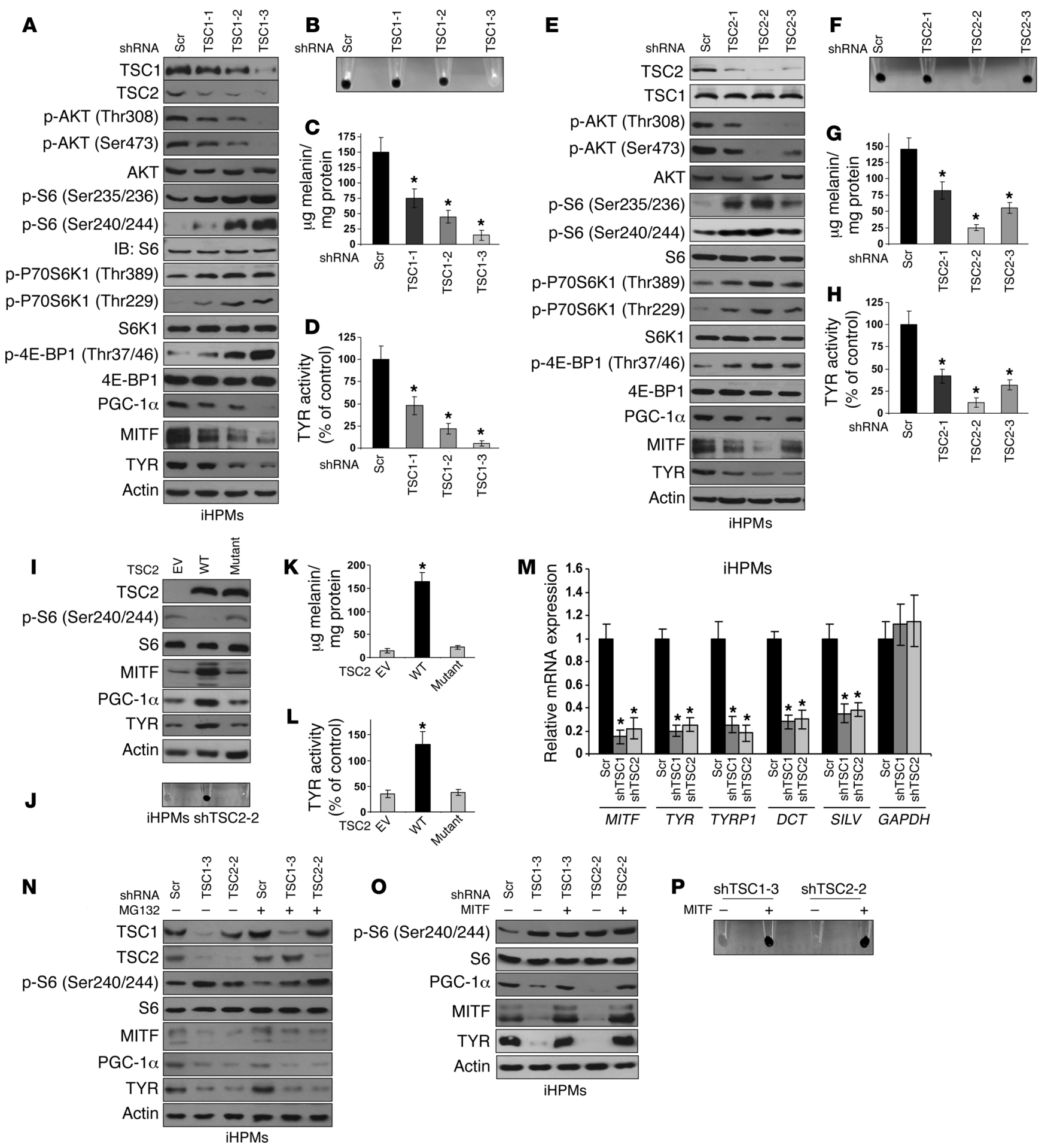

Figure 1. Disruption of the TSC protein complex induces loss of pigmentation in melanocytes. (A-D) Highly pigmented iHPMs were infected with lentiviruses expressing TSC1 shRNA or scrambled control (Scr) shRNA and selected with puromycin. (A) Immunoblot analysis shows that TSC1 depletion led to reduced MITF-M and its downstream targets PGC-1 $\alpha$ and TYR, as well as mTORC1 activation. (B) Cell pellets from TSC1-depleted cells had reduced pigmentation. (C and D) TSC1-depleted cells had reduced melanin and TYR activity. (E-H) Entirely similar to the data shown in A-D, except that TSC2 shRNAs were expressed. (I-L) TSC2-depleted iHPMs were reconstituted with lentiviruses containing WT TSC2 or patient-derived TSC2-mutant (P419S) or empty vector control viruses (EV) and selected with puromycin. (I) Immunoblot analysis shows that WT TSC2, but not the TSC2 mutant, rescued mTORC1 activation, MITF and PGC-1 $\alpha$ expression, and TYR protein levels. (J) Pigmentation, (K) melanin content, and (L) TYR activity were also all rescued by WT TSC2. Data in C, D, G, H, K, L, and $\mathbf{M}$ represent the mean $\pm S D$ from at least 3 independent experiments. ${ }^{*} P<0.05$, by 2-tailed Student's $t$ test. (M) Expression of the indicated genes was measured by qPCR in control or shRNA-expressing cells. GAPDH was used as a control. (N) Immunoblot analysis of lysates from TSC1- or TSC2-depleted cells treated with MG132 (25 $\mu$ M) for 6 hours. MITF, PGC-1 $\alpha$, and TYR were not rescued by MG132 treatment. ( $\mathbf{O}$ and $\mathbf{P})$ TSC1- or TSC2-depleted cells were infected with lentiviruses containing MITF-M or empty vector control (Scr) and selected with puromycin. (0) Immunoblot analysis shows increased MITF and TYR expression in cells infected with MTIF-M lentivirus. (P) Ectopic expression of MITF rescued the pigmentation loss in TSC1- and TSC2-depleted cells. The immunoblots presented in the figure panels include replicate samples run on parallel gels. 

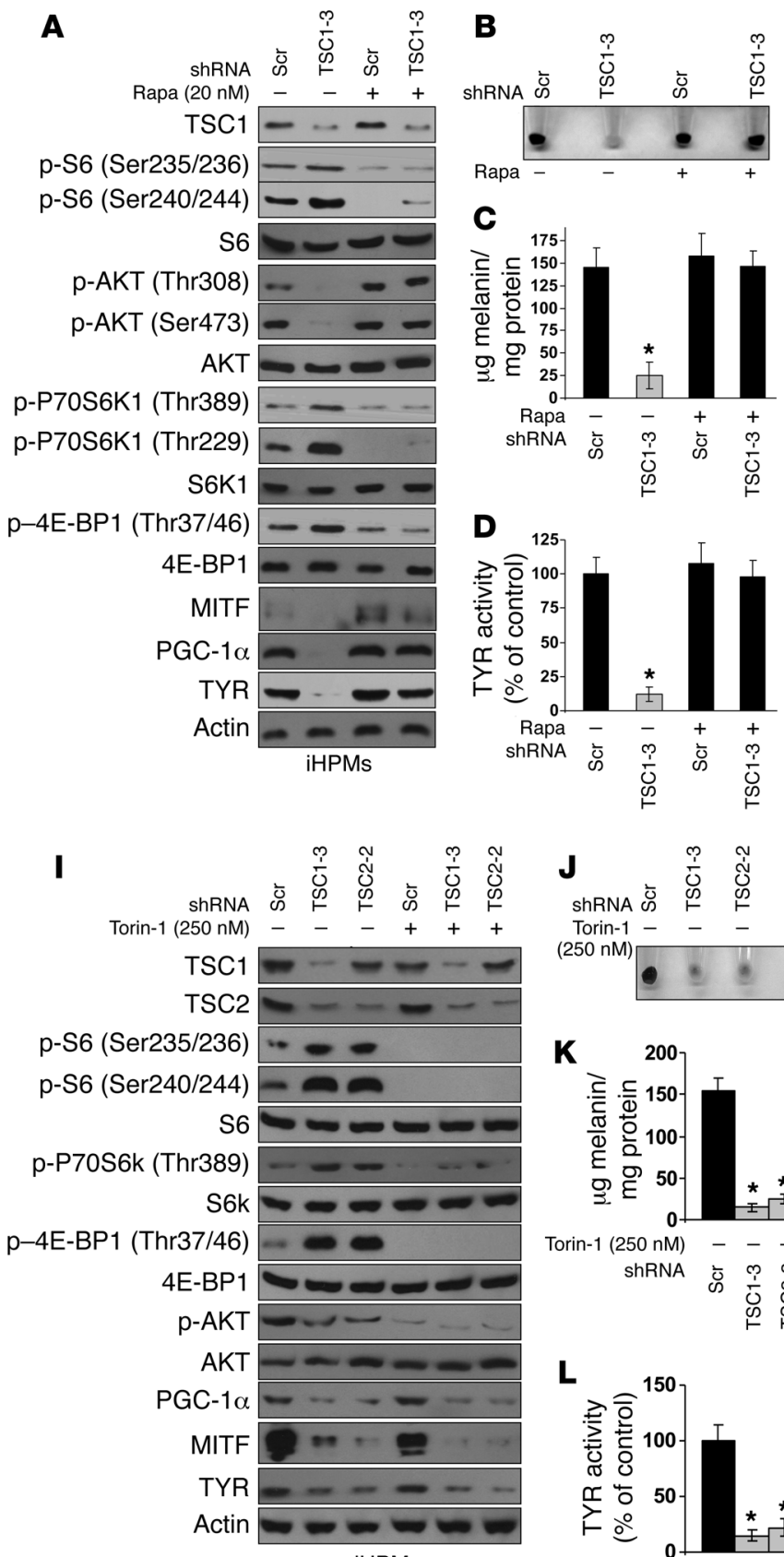

iHPMs

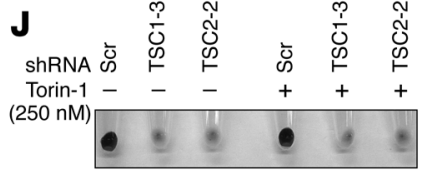

K

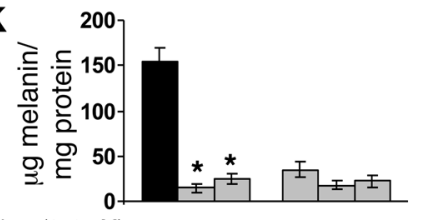

Torin-1 (250 nM) - - + + +

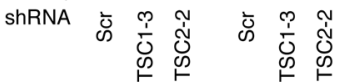

$\mathbf{L}$

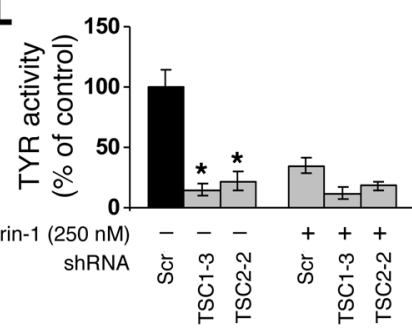

$\mathbf{E}$

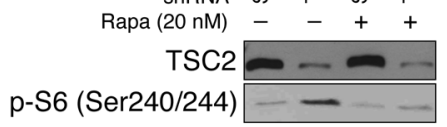

p-S6 (Ser235/236)

p-AKT (Thr308)

S6

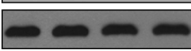

p-AKT (Ser473)

AKT

p-P70S6K1 (Thr389)

p-P70S6K1 (Thr229)

S6K1

$-0$

$4 \mathrm{E}-\mathrm{BP} 1 \sim--$

MITF

PGC-10

TYR

Actin

\section{F}

$-\infty$

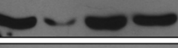

$---$

000

iHPMs

M
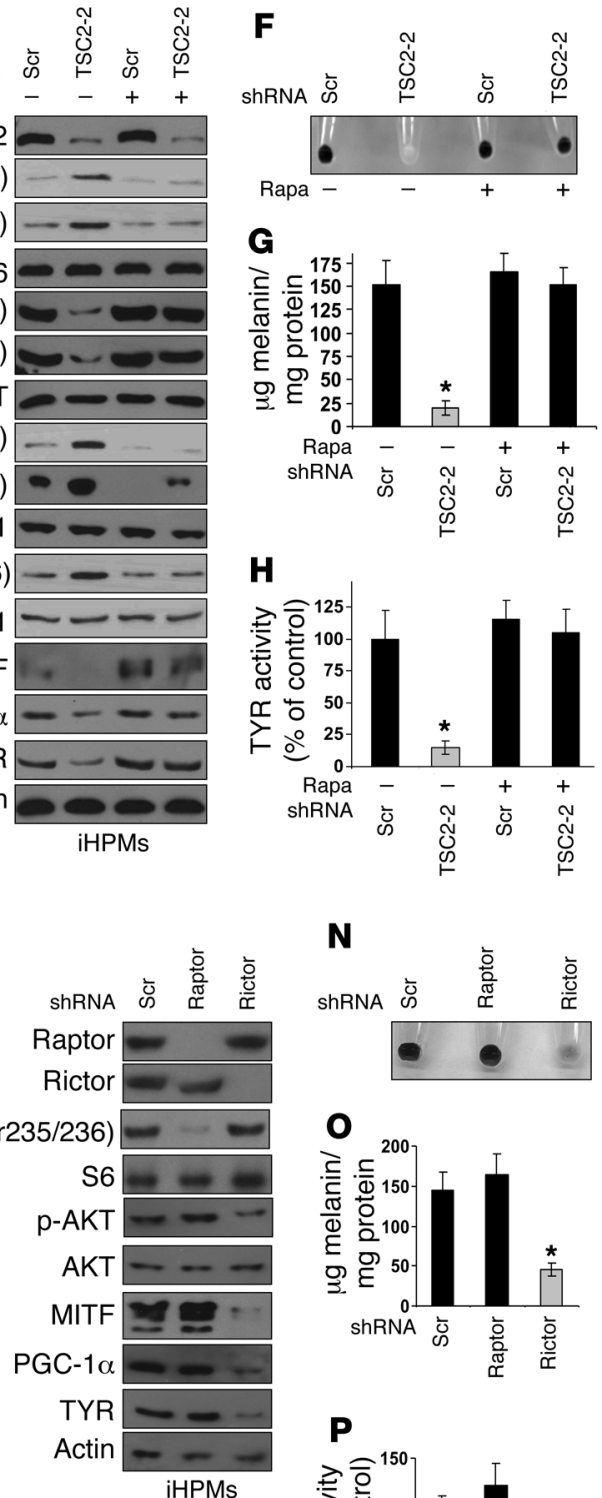

H

\section{$\mathbf{N}$}

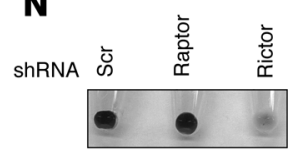

p-S6 (Ser235/236) - - -
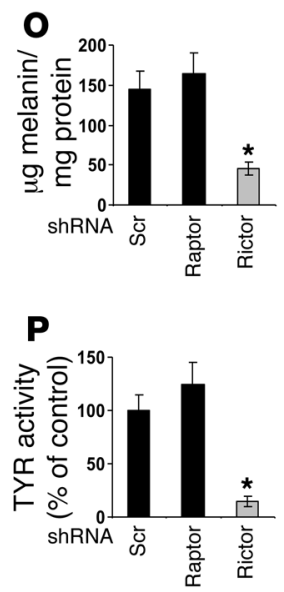

Figure 2. Rapamycin, but not Torin-1, restores the loss of pigmentation in TSC protein complex-deficient melanocytes. (A-D) iHPMs stably expressing shTSC1 or control (Scr) were treated with rapamycin (Rapa) (20 nM) or vehicle control (DMSO) for 72 hours. (A) Immunoblot analysis shows recovery of MITF expression, suppression of mTORC1, and recovery of p-AKT levels in cells treated with rapamycin. (B) Pigmentation, (C) melanin content, and (D) TYR activity were also all rescued by rapamycin. (E-H) Entirely similar to the data shown in A-D, except using iHPMs with shTSC2 knockdown. (I-L) iHPMs stably expressing shTSC1, shTSC2, or control (Scr) were treated with Torin-1 (250 nM) or vehicle control (DMSO) for 72 hours. (I) Immunoblot analysis shows complete suppression of mTORC1 and mTORC2. (J) Pigmentation, (K) melanin content, and (L) TYR activity, none of which were rescued by Torin-1 treatment. (M-P) iHPMs were infected with control (Scr) or shRaptor or shRictor lentiviruses and selected with puromycin for 6 days. (M) Immunoblot analysis shows that Rictor knockdown reduced MITF, PGC-1 $\alpha$, and TYR expression. (N) Pigmentation, $(\mathbf{O})$ melanin content, and (P) TYR activity, all of which show reduced pigmentation in Rictor-knockdown cells. Data in $\mathbf{C}, \mathbf{D}, \mathbf{G}, \mathbf{H}, \mathbf{K}, \mathbf{L}, \mathbf{O}$, and $\mathbf{P}$ represent the mean \pm SD from at least 3 independent experiments. ${ }^{*} P<0.05$, by Student's $t$ test. The immunoblots presented in the figure panels include replicate samples run on parallel gels. 
treated the cells with the catalytic mTORC1/2 inhibitor Torin-1 (56). Interestingly, in contrast to rapamycin, Torin-1 was not able to restore MITF expression, its target genes PGC- $1 \alpha$ or TYR, or pigmentation in either TSC1- or TSC2-knockdown cells (Figure 2, I-L, and Supplemental Figure 2, I-L), even though it completely inhibited mTORC1 as assessed by loss of phosphorylation of S6, S6K1, and 4E-BP1 (Figure 2I and Supplemental Figure 2I). We suspected that inhibition of MTORC 2 by Torin-1, indicated by the loss of phosphorylation of AKT (Figure 2I and Supplemental Figure 2I), accounted for this difference. To examine that possibility further, we knocked down Raptor and Rictor, which are essential and unique components of mTORC1 and mTORC2, respectively, using shRNA in both iHPMs and SK-MEL-30 cells. Depletion of Raptor had the expected effects on Raptor expression and reduced levels of the marker of mTORC1 activity, p-S6 (Ser235/236). Similarly, shRictor had the expected effects on Rictor levels and reduced p-AKT (Ser473) levels, indicating blockade of mTORC2 activity. Inhibition of mTORC2 by Rictor depletion was associated with a marked reduction in the expression of MITF and its target genes PGC-1 $\alpha$ and TYR, as well as reduced melanin production and pigmentation formation in iHPMs and SK-MEL-30 cells (Figure 2, $\mathrm{M}-\mathrm{P}$ and Supplemental Figure 2, M-P). In contrast, inhibition of mTORC1 by shRaptor did not reduce pigmentation, but marginally increased MITF expression (Figure 2, M-P, and Supplemental Figure 2, M-P). In summary, these results indicated that rapamycin, but not Torin-1, rescued the reduced pigmentation seen in melanocytes lacking the TSC complex and that loss of Rictor had effects similar to those seen with loss of the TSC complex in reducing pigmentation. In aggregate, these findings suggested that the reduced AKT activity observed with loss of both the TSC complex and Rictor might be responsible for the reduction in pigment biosynthesis.

TSC protein complex controls melanogenesis through stabilization of $\beta$-catenin. Because MITF expression is controlled at multiple levels, we considered 2 possible mechanisms for the reduction of MITF in response to loss of the TSC protein complex and downstream mTORC1 activation in these cells: a) accelerated degradation of MITF due to phosphorylation or other events secondary to mTORC1 activation; and b) altered transcriptional regulation. To examine the possibility of accelerated MITF degradation, we used the proteasome inhibitor MG132 (at $25 \mu \mathrm{M}$ ) to treat cells expressing the most efficient TSC1 and TSC2 shRNAs. In both iHPMs and SK-MEL-30 cells, MG132 treatment did not restore the expression of MITF, PGC- $1 \alpha$, or TYR (Figure $1 \mathrm{~N}$ and Supplemental Figure 1N). As an internal control, TSC2 levels were increased in the MG132-treated TSC1-knockdown cells, indicating effective proteasome blockade. Thus, accelerated MITF degradation did not appear to contribute to the reduction in MITF levels. Second, to explore whether altered transcriptional cues following loss of the TSC complex alters MITF levels, we examined AKT activation and the effects on its downstream substrate GSK3 $\beta$ $(57,58)$. We observed a marked reduction in p-AKT (Thr308), p-AKT (Ser473), and p-GSK3 $\beta$ (Ser9) levels in iHPMs with knockdown of either TSC1 or TSC2 (Figure 3A), with similar findings in SK-MEL-30 cells (Supplemental Figure 3A). As noted above, free GSK3 $\beta$ is regulated by AKT and is also present in cells as an essential component of the APC destruction complex that drives $\beta$-catenin degradation (59). Since increased levels of active GSK3 $\beta$ would be expected to lead to phosphorylation and degradation of $\beta$-catenin, reducing nuclear $\beta$-catenin-TCF/LEF complex levels to reduce MITF transcription $(42,60,61)$, we examined $\beta$-catenin levels. Knockdown of either TSC1 or TSC2 led to increased levels of $\mathrm{p}-\beta$-catenin (Ser33/37 and Thr41) and reduced levels of $\beta$-catenin in iHPMs and SK-MEL-30 cells (Figure 3A and Supplemental Figure 3A). Furthermore, rapamycin treatment of the knockdown cells decreased $p-\beta$-catenin and increased $\beta$-catenin levels (Figure $3 \mathrm{~B}$ and Supplemental Figure $3 \mathrm{~B}$ ), suggesting that this effect was due to mTORC1 activity. The nuclear fraction of $\beta$-catenin was markedly diminished in knockdown cells to a greater extent than was seen with total levels, consistent with a consequent lack of transcriptional activity (Figure 3C and Supplemental Figure 3C), and nuclear levels of $\beta$-catenin were also restored by rapamycin treatment (Figure 3D and Supplemental Figure 3D).

To examine the effects of TSC complex loss on MITF transcription in greater detail, we expressed an MITF-luciferase reporter construct (42). MITF promoter activity was significantly reduced in both iHPMs and SK-MEL-30 cells with either TSC1 or TSC2 knockdown and was fully restored by treatment with rapamycin (Figure 3G and Supplemental Figure 3G). In addition, mRNA levels of MITF and its target TYR were considerably diminished by TSC1 or TSC2 knockdown and were restored to higher-than-normal levels by treatment with rapamycin (Figure $3 \mathrm{H}$ and Supplemental Figure $3 \mathrm{H}$ ). To confirm that loss of nuclear $\beta$-catenin had transcriptional effects that would lead to loss of MITF expression, we used the TK-TOP reporter in parallel assays (62). We observed that TSC1/TSC2-deficient cells had a 5-fold reduction of TCF/LEF transcriptional activity (Figure 3E and Supplemental Figure 3E). These experiments suggested that $\beta$-catenin-TCF/LEF transcriptional activity is markedly attenuated in melanocytes with TSC1/ TSC2 loss, explaining the marked reduction of MITF mRNA levels. Rapamycin treatment fully restored $\beta$-catenin-TCF/LEF transcriptional activity (Figure 3F and Supplemental Figure 3F), indicating that this series of events was due to aberrant mTORC1 activation.

To confirm that changes in $\beta$-catenin expression and nuclear localization were the main driver of reduced MITF expression in iHPMs and SK-MEL-30 cells, we examined the effects of expression of WT and constitutively active (CA) mutant (S33Y) $\beta$-catenin. WT and CA $\beta$-catenin expression in both TSC1- and TSC2-knockdown iHPMs and SK-MEL-30 cells led to increased expression of MITF, PGC- $1 \alpha$, and TYR, as well as a return of pigmentation (Figure 4, A-D and Supplemental Figure 4, A-D).

TSC protein complex regulates $\beta$-catenin by inhibition of GSK3 $\beta$. Guided by previous studies linking GSK3 $\beta$ with both AKT and $\beta$-catenin, we next sought to explain the mechanism by which reduced AKT activity affected $\beta$-catenin levels. To directly assess the role of GSK3 $\beta$, we used 2 approaches. First, shRNA-mediated GSK3 $\beta$ depletion within cells with suppressed TSC1 or TSC2 markedly reduced the phosphorylation of $\beta$-catenin at Ser33/37 and Thr41 and restored total $\beta$-catenin protein to levels similar or higher than those seen in control cells (Figure 4E, and Supplemental Figure 4E). Furthermore, expression of MITF and its target genes PGC-1 $\alpha$ and TYR was significantly increased at both RNA and protein levels, as was MITF promoter activity and pigment formation in the shRNA-GSK3 $\beta$-derivative cell lines (Figure 4, E-H and Supplemental Figure 4, E-H). In addition, reduced 

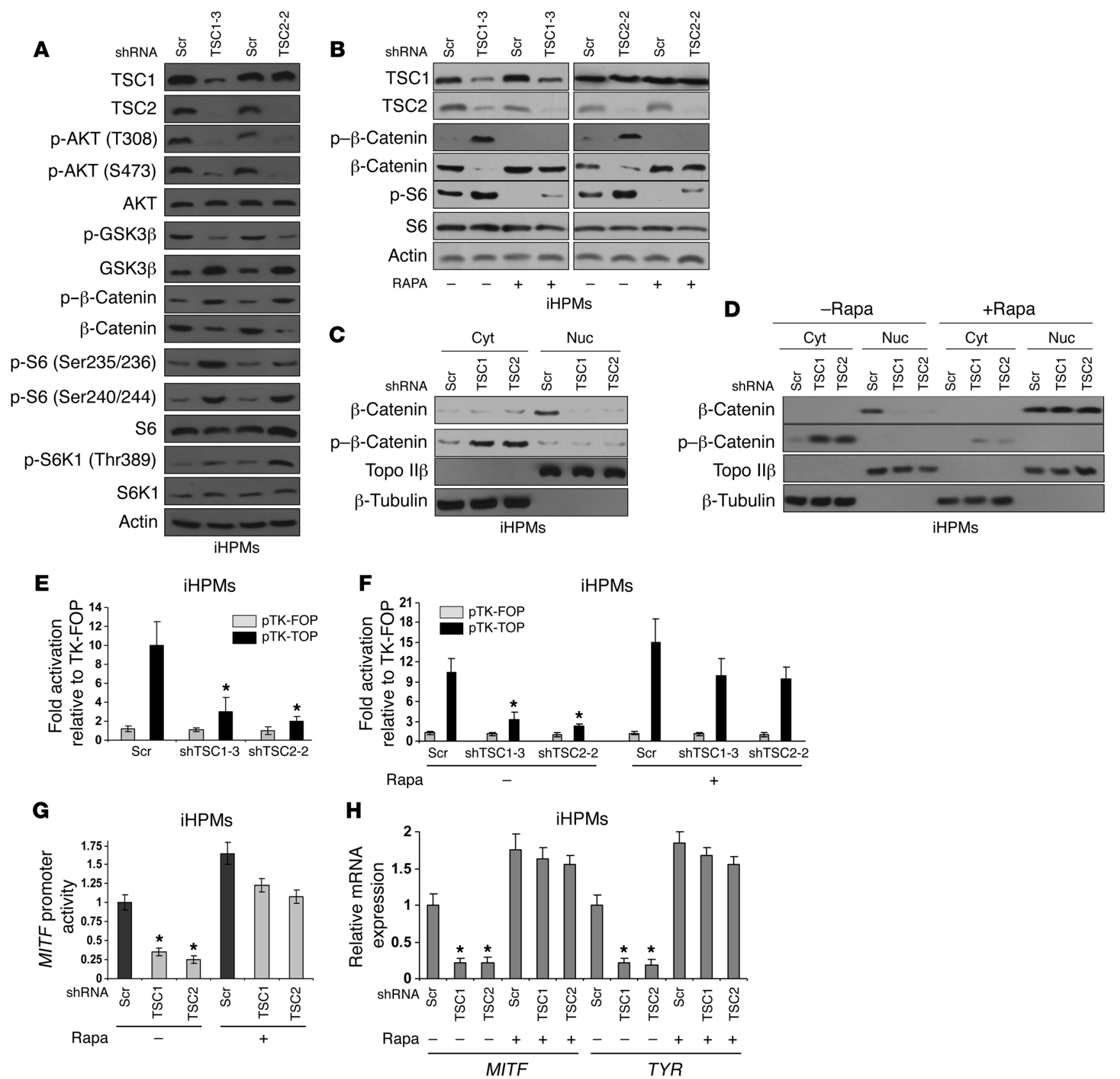

Figure 3. Disruption of the TSC protein complex leads to GSK3 $\beta$ activation and reduced $\beta$-catenin and MITF transcription in human primary melanocytes. (A) Immunoblot analysis of iHPMs expressing shTSC1 or shTSC2 or control (Scr) shows reduced p-AKT (Ser473/Thr308), reduced p-CSK3 $\beta$ (Ser9), increased CSK3 $\beta$, reduced $\beta$-catenin and increased p- $\beta$-catenin (Ser33/Ser37/Thr41). (B) Immunoblot analysis of iHPMs expressing shTSC1 or shTSC2 or control (Scr), treated with rapamycin ( $20 \mathrm{nM}$ ) or vehicle (DMSO) for 72 hours shows recovery of $\beta$-catenin levels. (C) Immunoblot analysis of cytosolic (Cyt) and nuclear (Nuc) fractions shows loss of nuclear $\beta$-catenin in the TSC1- and TSC2-knockdown cells. Topo II $\beta$ and $\beta$-tubulin served as controls for the nucleus and cytosol, respectively. (D) Entirely similar to the data shown in C, except that cells on the right were treated with rapamycin for 4 days, and $\beta$-catenin was seen again in the nuclear fraction. (E) Relative luciferase expression in iHPMs stably expressing shTSC1 or shTSC2 or control (Scr), transfected with TK-TOP- or TK-FOP-driven luciferase. (F) Entirely similar to the data shown in E, except that iHPMs were treated with rapamycin (20 nM) or vehicle (DMSO) for 72 hours prior to analysis. (C) MITF promoter activity of iHPMs with TSC1 or TSC2 knockdown, treated with or without $20 \mathrm{nM}$ rapamycin and measured using pCL3-luciferase empty vector or MITF-PGL3-luciferase. (H) Cells treated as in $\mathbf{G}$ were analyzed by qPCR for MITF and TYR mRNA levels. Data in E-H represent the mean \pm SD from at least 3 independent experiments. ${ }^{*} P<0.05$, by Student's $t$ test. The immunoblots presented in the figure include replicate samples run on parallel gels.

GSK3 3 expression had no effect on mTORC1 activation, measured as p-S6, in the TSC1/TSC2-knockdown cells (Figures 4E, and Supplemental Figure $4 \mathrm{E}$ ), indicating that this effect was independent of mTORC1. These results suggested that hypophosphorylated GSK3 $\beta$ was phosphorylating $\beta$-catenin, leading to its destruction.
As a second approach to confirm the role of GSK3 3 in the regulation of $\beta$-catenin in these melanocyte cell lines, we used pharmacological inhibitors specific to GSK3ß. Using the pan-GSK3 inhibitor CHIR-99021 (63-65), we observed effects similar to those of shRNA against GSK3ß. CHIR-99021 treatment reduced levels of 


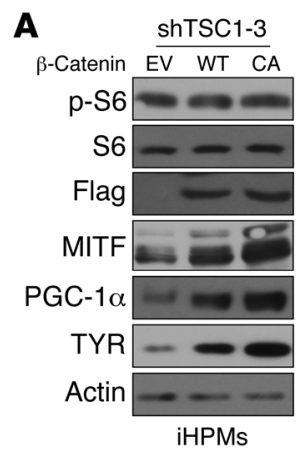

C

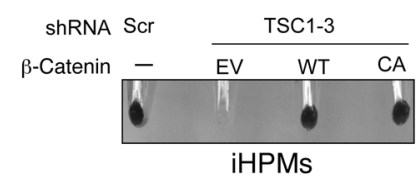

D

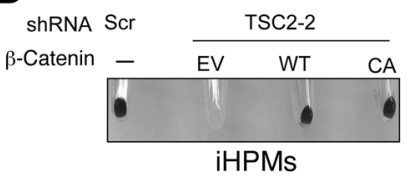

E

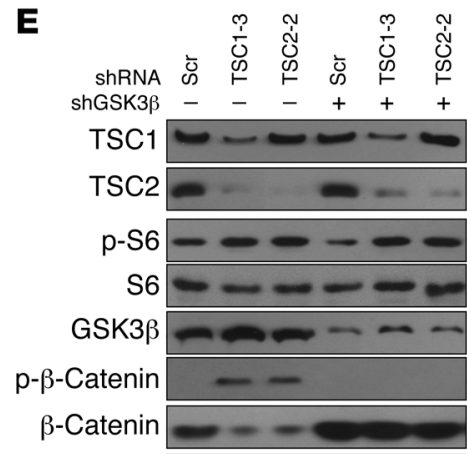

PGC-1 $\alpha-\infty-\infty \omega$

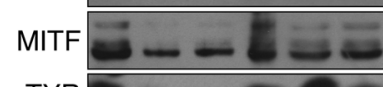

TYR

Actin

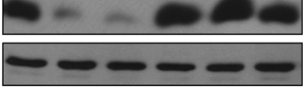

iHPMs

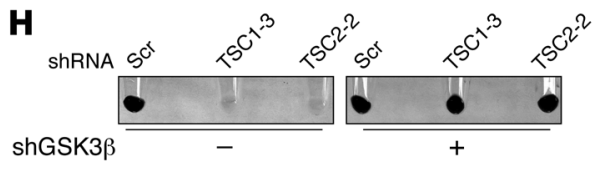

iHPMs

Figure 4. Ectopic expression of $\beta$-catenin or knockdown of GSK3 $\beta$ restores pigmentation in TSC protein complex-depleted melanocytes. (A) Immunoblot analysis of iHPMs stably expressing shTSC1 or shTSC2 transduced to express WT $\beta$-catenin or CA $\beta$-catenin (S33Y) or empty vector (EV) control. Note the expression of MITF in WT and CA $\beta$-catenin-expressing iHPMs. (B) Entirely similar to the data shown in A, except that iHPMs expressed shTSC2. (C and $\mathbf{D}$ ) Pigmentation of cells from $\mathbf{A}$ and B, respectively. (E) Immunoblot analysis of iHPMs stably expressing shTSC1 or shTSC2 transduced to express GSK3 $\beta$ shRNA or control shRNA. Note the marked enhancement of $\beta$-catenin expression in the GSK3 $\beta$ shRNA cells. (F) Luciferase assay performed on the cells in $\mathbf{E}$ after transfection with pCL3-luciferase empty vector or MITF-PGL3-luciferase. (C) qPCR analysis of MITF and TYR mRNA levels in the cells described in E shows marked enhancement of MITF and TYR expression by GSK3 $\beta$ shRNA. (H) Pigmentation of cells from $\mathbf{E}$. Data in $\mathbf{F}$ and $\mathbf{G}$ represent the mean \pm SD from at least 3 independent experiments. ${ }^{*} P<0.05$, by Student's $t$ test. The immunoblots presented in the figure include replicate samples run on parallel gels.

$\mathrm{p}-\beta$-catenin (Ser33/37 and Thr41) and increased both total and nuclear $\beta$-catenin levels in both control and TSC1/TSC2-depleted SK-MEL-30 and iHPM cell lines (Figure 5, A and D, and Supplemental Figure 5, A and B). Corresponding to this increase in $\beta$-catenin levels, CHIR-99021 treatment reversed the effects induced by TSC protein complex depletion on: mRNA and protein levels of MITF and its downstream targets PGC-1 $\alpha$ and TYR (Figure 5A, Figure 6B, and Supplemental Figure 5, A and D); MITF promoter activity (Figure 6A and Supplemental Figure 5C); and pigment formation (Figure 5F and Supplemental Figure 5E). To confirm that these effects of the inhibitor were specific to effects on GSK3 $\beta$, we tested 2 other structurally unrelated GSK3 $\beta$-specific inhibitors, AR-A014418 $(66,67)$ and 6-bromoindirubin-3'-oxime (BIO) (68), and found that both were identical in their ability to reverse the effects of loss of the TSC protein complex (Figure 5, B, C, E, G, and H, Figure 6, C-F, and Supplemental Figure 5, F-N).

Taken together, our data demonstrate that in melanocytes and melanoma cells, the TSC protein complex suppresses mTORC1 signaling, leading to physiological activity of $\mathrm{MTORC} 2$ and AKT, inhibition of GSK3 $\beta$, stabilization and nuclear transport of $\beta$-catenin to activate the TCF/LEF1 transcription complex to cause transcription of MITF, production of MITF's downstream melanogenic genes, and production of pigment.

mTORC1 is hyperactive in melanocytes from TSC patients' hypomelanotic macules due to loss of TSC complex activity. Hav- ing established a mechanistic connection between normal TSC complex activity and pigmentation in melanocytes in vitro, we examined our hypothesis that spontaneous loss of the TSC complex occurred in hypomelanotic macules from patients with TSC. Skin biopsy samples were obtained from 6 hypomelanotic macules from 7 patients with TSC, with nearby normally pigmented skin available from 5 of these patients. Primary melanocyte and fibroblast cultures were prepared. Since these were primary cell cultures without immortalization, the melanocytes grew for a variable period of time prior to the onset of senescence, which limited the amounts of material available for various studies.

Melanocytes cultured from the hypomelanotic macules had features consistent with those observed in our studies of TSC1and TSC2-knockdown iHPM and SK-MEL-30 cell lines (Figure $7 \mathrm{~A}$ and Supplemental Figure 6A). In comparison with normal skin melanocytes, hypomelanotic macule melanocyte cultures showed a marked reduction in TSC2, but not TSC1, protein expression (Figure 7A and Supplemental Figure 6A). mTORC1 was activated, as assessed by phosphorylation of its downstream targets S6, S6K1, and 4E-BP1 (Figure 7A and Supplemental Figure 6A). AKT activity was reduced, as assessed by reduced phosphorylation at Thr308 and Ser473. Levels of p-GSK3 $\beta$ were reduced, while total GSK3 $\beta$ levels were increased, and $\mathrm{p}-\beta$-catenin was increased, while total $\beta$-catenin was markedly reduced (Figure 7A and Supplemental Figure 6A). Protein levels of MITF and its target genes PGC-1 $\alpha$ 

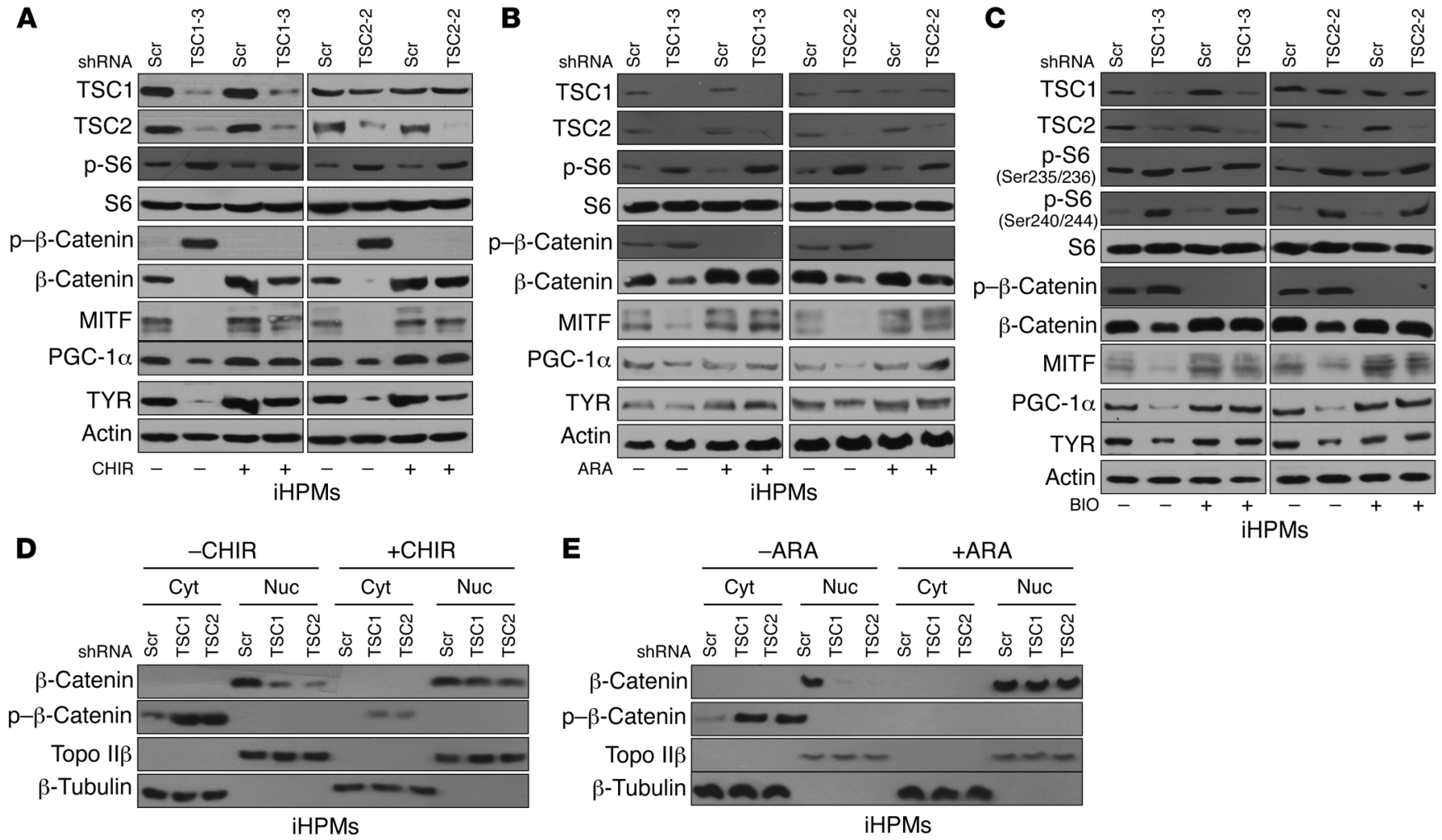

iHPMs

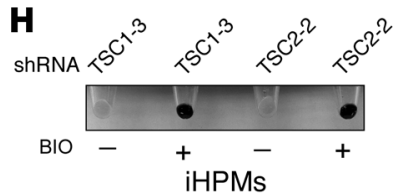

Figure 5. GSK3 $\beta$ inhibition prevents loss of pigmentation in response to TSC protein complex loss. (A) Immunoblot analysis of iHPMs expressing shTSC or shTSC2 or control (Scr) after treatment with the GSK3 $\beta$-specific inhibitor CHIR-99021 (CHIR) (3 $\mu$ M) for 5 days. Note the recovery of $\beta$-catenin and MITF expression in cells treated with CHIR-99021. (B) Entirely similar to the data shown in A, except using the GSK3 3 -specific inhibitor AR-A014418 (ARA) $(5 \mu \mathrm{M})$ for 5 days. (C) Entirely similar to the data shown in A, except using the GSK3 $\beta$-specific inhibitor 6-bromoindirubin-3'-oxime (BIO) (2 $\mu \mathrm{M})$ for 5 days. (D and E) Immunoblot analysis shows recovery of nuclear $\beta$-catenin in iHPMs following treatment with CHIR-99021 and AR-A014418, respectively. (F-H) Pigmentation was restored in cells treated with CHIR-99021, AR-A014418, and BIO, respectively. The immunoblots presented in the figure include replicate samples run on parallel gels.

and TYR were markedly reduced (Figure 7A and Supplemental Figure 6A). Pigmentation was also markedly reduced in melanocytes from the hypomelanotic macules, in contrast to those from normal skin (Figure 7B and Supplemental Figure 6B).

We also examined mRNA levels of TSC1, TSC2, MITF, and MITF target genes in 5 of these cultures from which there was sufficient material. In comparison with normal skin melanocytes, hypomelanotic macule melanocytes showed markedly reduced expression of TSC2, MITF, and several downstream MITF target genes (Figure 8C).

In contrast, fibroblasts cultured from 2 of the hypomelanotic macules showed no difference in TSC1 or TSC2 expression and had normal regulation of mTORC1 activity (Figure 7D), indicating that the defect in TSC2 expression and the downstream effects were specific to the melanocytes.

We analyzed all melanocyte cultures for mutations in TSC1/ $T S C 2$. Five of six patients carried a mutation in TSC2, while in one patient with two specimens, no mutation in either TSC1 or TSC2 could be identified. The occurrence of a germline mutation in TSC2 in 5 of 6 patients was concordant with the observation that TSC2 expression was reduced, while TSC1 expression was normal, in all of these hypomelanotic macule melanocyte cultures.

One hypomelanotic macule melanocyte culture (P35) had 2 mutations in TSC2: c.2098-1G>A and c.1595-1597delAGA p.(Lys533del). The fibroblast culture from the same biopsy showed only the TSC2 c.2098-1G>A mutation, consistent with its occurrence as a germline event. These observations clearly imply that the c.1595-1597delAGA p.(Lys533del) mutation was a somatic mutation, occurring only in the hypomelanotic macule melanocytes. However, no second mutations were seen in any of the other melanocyte cultures.

We also analyzed the expression of MITF and the phosphorylation of S6 in paraffin-embedded skin biopsy samples from 2 TSC patients' hypomelanotic macules and nearby normal skin. Normal skin had melanin (brown) in the basal layer of the epidermis, including melanocytes, which were identified by strong MITF 
A
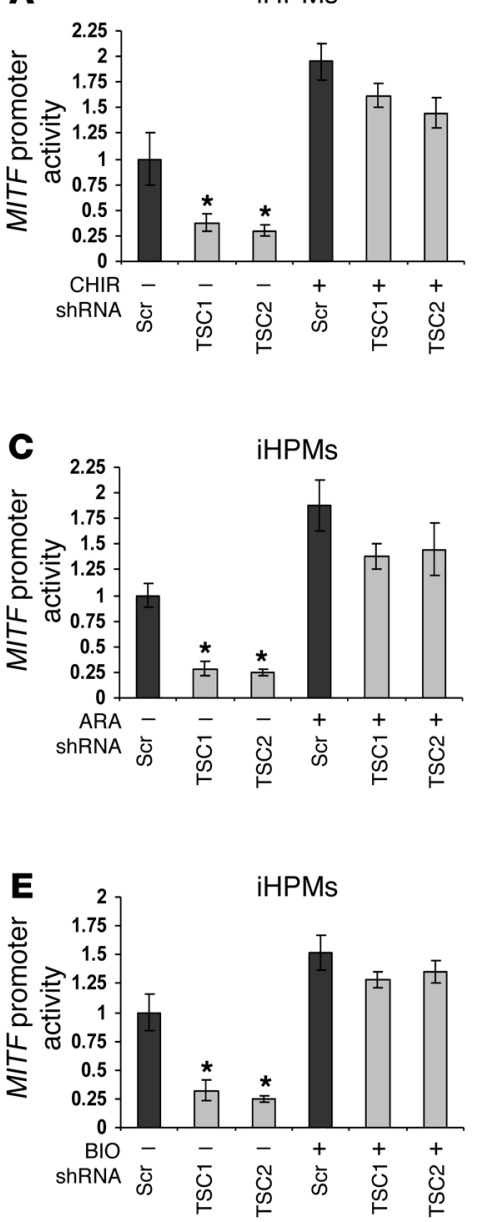

B
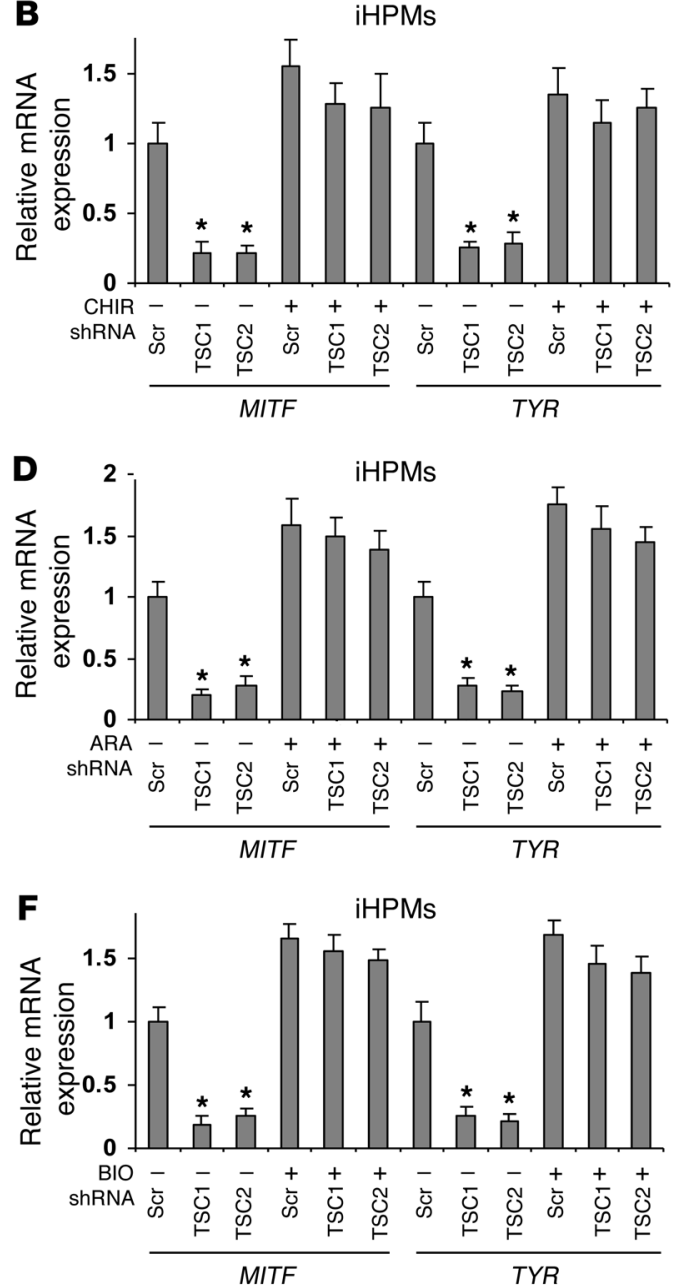

Figure 6. GSK3 $\beta$ inhibition restores MITF transcriptional activity in response to TSC protein complex loss. (A) MITF promoter activity of iHPMs with TSC1 or TSC2 knockdown, treated with or without $3 \mu \mathrm{M}$ CHIR99021 and measured using pCL3-luciferase empty vector or MITF-PGL3-luciferase. (B) Cells treated as in $\mathbf{A}$ were analyzed by qPCR for MITF and TYR mRNA levels. (C and D) Entirely similar to the data shown in $\mathbf{A}$ and B, except using the GSK3 $\beta$-specific inhibitor AR-A014418 $(5 \mu \mathrm{M})$ for 5 days. ( $E$ and $\mathbf{F}$ ) Entirely similar to the data shown in $\mathbf{A}$ and B, except using the GSK3 $\beta$-specific inhibitor 6-bromoindirubin-3'-oxime (BIO) $(2 \mu \mathrm{M})$ for 5 days. Data in $\mathbf{A}-\mathbf{F}$ are presented as the mean \pm SD from at least 3 independent experiments. ${ }^{*} P<0.05$, by Student's $t$ test. expression (Figure 7C, top left). The hypomelanotic macule biopsy showed much less melanin and somewhat reduced expression of MITF (Figure 7C, bottom left). Furthermore, melanocytes in the hypomelanotic macule had increased p-S6 and reduced MelanA (Figure 7C, bottom right) compared with melanocytes from normal skin (Figure 7C, top right).

To further characterize the TSC2-deficient melanocytes, we examined the cultured cells by transmission electron microscopy (TEM). TEM sections showed that melanocytes from the hypomelanotic macules of 3 patients accumulated fewer pigmented melanosomes than did control skin (from 2 patients, Figure 8A). Furthermore, blinded melanosome grading and counting of multiple sections of melanocytes showed that hypomelanotic macules had a reduced proportion of mature, stage-IV melanosomes and a higher proportion of immature stage-I melanosomes (Figure 8B).

In aggregate, these results are consistent with our hypothesis that TSC hypomelanotic macules occur as a result of secondary events leading to a near-complete loss of TSC1/TSC2 during a melanoblast-to-melanocyte differentiation that results in clonal skin patches containing immature melanocytes with mTORC1 activation and reduced MITF-dependent pigment production. The lack of distinguishable somatic mutations in the majority of the cultures suggests that hitherto unidentified epigenetic mechanisms may occur as a "second hit" in a significant frac- tion of hypomelanotic macules and thus result in monoallelic expression of mutant TSC2.

\section{Discussion}

TSC is a tumor-suppressor gene syndrome with diverse manifestations, many of which are tumors that occur in multiple organ systems including brain, skin, kidneys, heart, and lungs $(1,69)$. Although TSC tumors are typically histologically benign, their progressive growth in the brain, kidneys, or lungs can lead to severe consequences including death, if not managed properly. Historically, surgery has been used as the primary treatment approach for TSC tumors, but recent clinical trials have shown that rapalog therapy has clinical benefit in controlling the growth of TSC-related tumors (70-74).

Here, we present evidence that a functional TSC complex is required for normal pigment formation by melanocytes both in vitro and in vivo (modeled in Figure 9). We demonstrate that loss of the TSC protein complex, through loss of either of its constituent proteins, TSC1 or TSC2, leads to GSK3 $\beta$ activation, with a consequent loss of nuclear $\beta$-catenin and a marked reduction in MITF transcription and expression, resulting in loss of pigmentation. The conclusion that loss of TSC1 or TSC2 results in reduced pigmentation is supported by a recent clinical study, in which topical rapamycin treatment restored the pigmentation of TSC hypomelanotic macules (25). Our findings support the model that 


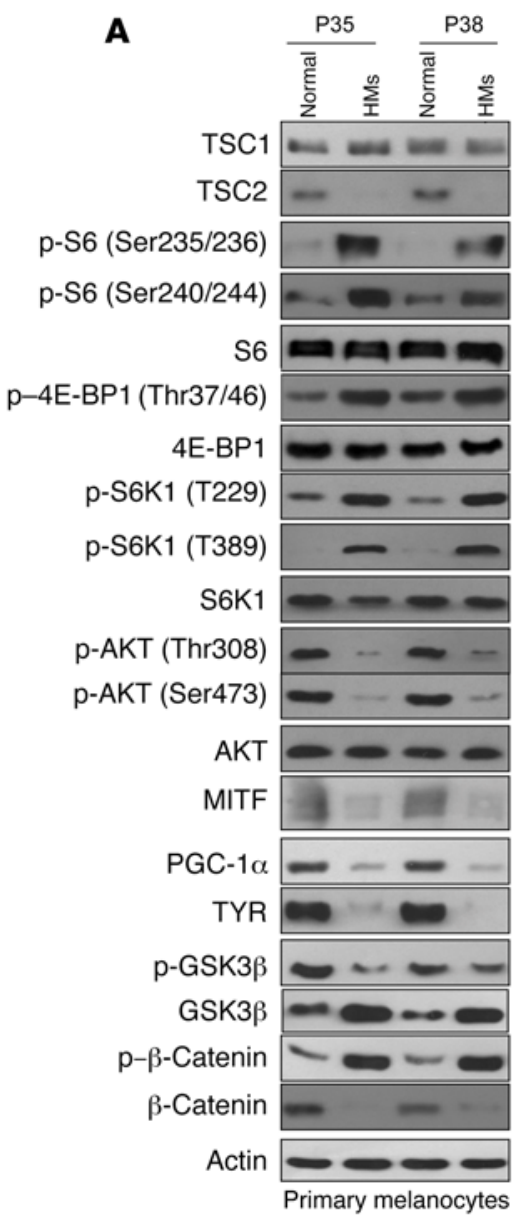

B

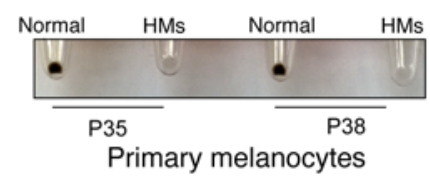

C

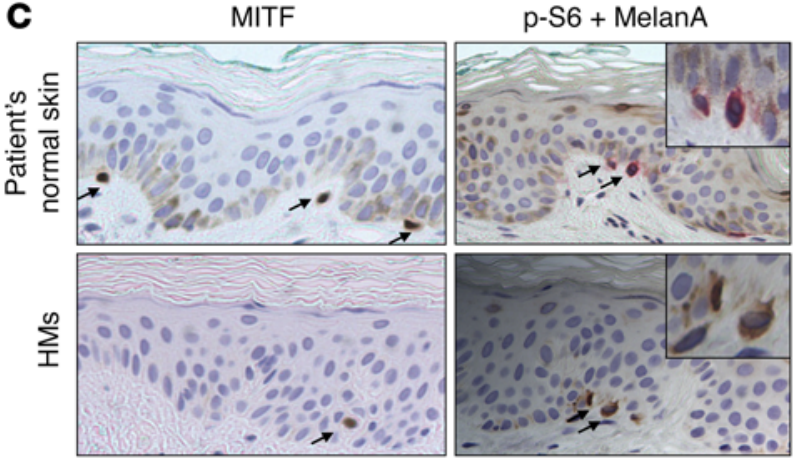

D

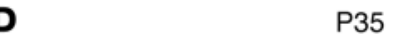

$\frac{\text { P38 }}{\text { Normal HM }}$

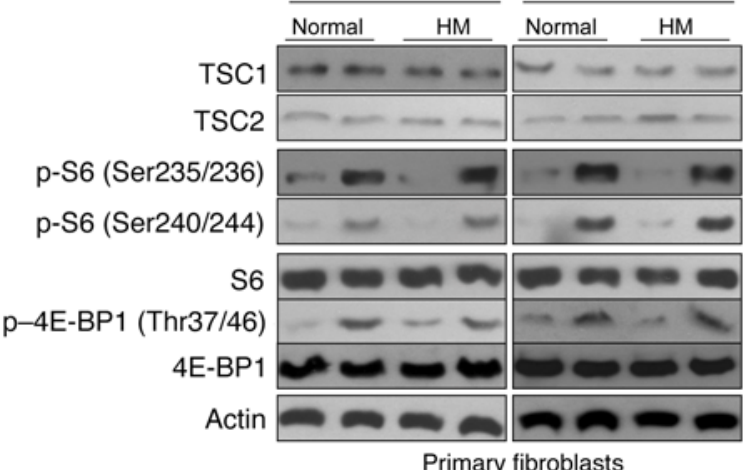

Primary fibroblasts

Figure 7. mTORC1 is hyperactive in melanocytes isolated from TSC patients' hypomelanotic macules. (A) Immunoblot analysis of primary melanocytes isolated from hypomelanotic macules (HMs) and nearby normal skin from TSC patients P35 and P38. Note the activation of mTORC1 in melanocytes from the hypomelanotic macules, with reduced MITF, PGC-1 $\alpha$, TYR, and $\beta$-catenin and increased GSK3 $\beta$. (B) Pigmentation of the melanocytes analyzed in A. (C) Immunohistochemical staining of TSC hypomelanotic macules and nearby normal skin. Note that melanin was present in the basal layer of the epidermis of normal skin (faint brown stain) and was reduced in HM skin. MITF staining in HM melanocytes was also somewhat reduced. Normal skin melanocytes had higher expression of MelanA and reduced expression of p-S6 compared with HM skin melanocytes. Original magnification, $\times 200$ and $\times 400$ (insets). (D) Immunoblot analysis of HM and normal skin fibroblast cultures from the same TSC patients studied in A and B. Immunoblots show similar regulation of mTORC1 in both sets of cells in response to serum deprivation and addback. The immunoblots presented in the figure include replicate samples run on parallel gels.

second-hit loss of TSC1 or TSC2, corresponding to the germline mutation present in an individual with TSC, occurs in melanoblast lineage cells during embryonic development and leads to clonal patches of hypopigmented skin. This model is supported by the clinical observation that TSC hypomelanotic macules can be observed at birth through the use of UV illumination, which led to their use as a reliable and early marker of TSC for decades $(22,24,75)$. This 2-hit mechanism is analogous to that underlying café-au-lait macules in neurofibromatosis, in which the melanocytes sustain 2 mutations in NF1 (76). The timing of the second-hit event leading to clonal patches in TSC may determine the size and shape of the hypopigmented skin, predicted to be earlier for the occasional large, segmental lesions and later for scattered small, confetti-like lesions. These patterns resemble the results of developmental studies in mice showing that single-cell-derived clonal melanocyte clusters occupy patches of skin in the mouse (49). Our results also suggest that, in melanocytes, TSC protein complex loss is accompanied by relatively little, if any, proliferative effect, consistent with the relative absence of TSC1/TSC2 mutations observed in melanoma genome-sequencing studies (77). Hence, these observations indicate that TSC complex loss does not lead to a proliferative advantage in all cell types, but rather that its effects are cell context dependent, helping explain the limited distribution and distinctive pathology of TSC-related tumors.

We were surprised to discover that only 1 of 7 hypomelanotic macule melanocyte cultures examined showed a second somatic mutation in TSC1 or TSC2. Four of six other patients' cultures showed a single mutation in TSC2, which was consistent with the reduced expression of TSC2 by those cells. To examine whether an altered epigenetic status could explain the nearly completely extinguished expression of TSC2 in these cultures, we performed bisulfite treatment, followed by sequencing of the TSC2 CpGrich promoter region, but found no evidence of $\mathrm{CpG}$ methylation. We suspect that long-range epigenetic or chromatin-mediated 
A

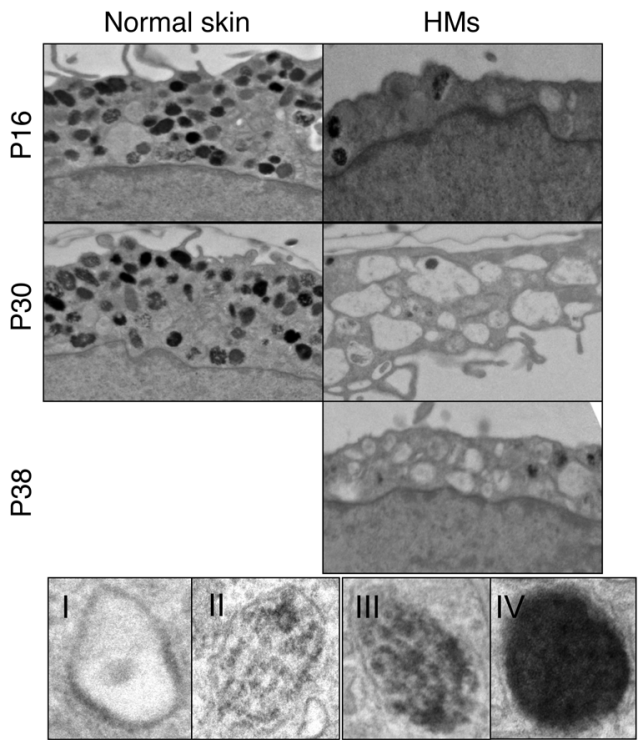

B

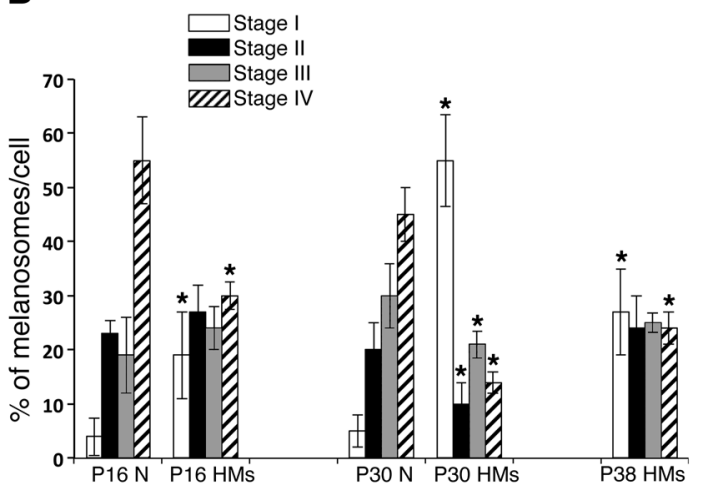

C

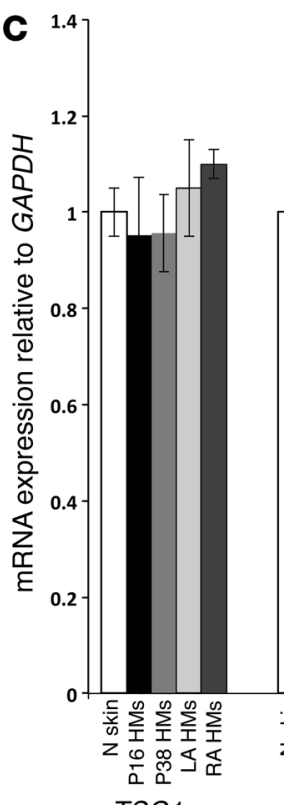

TSC1

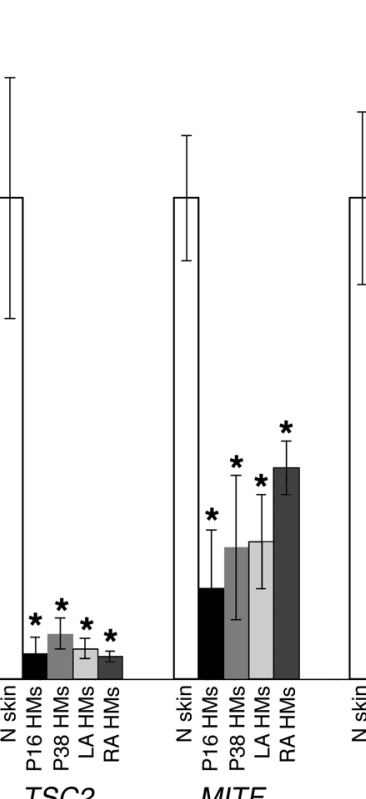

TSC2
MITF

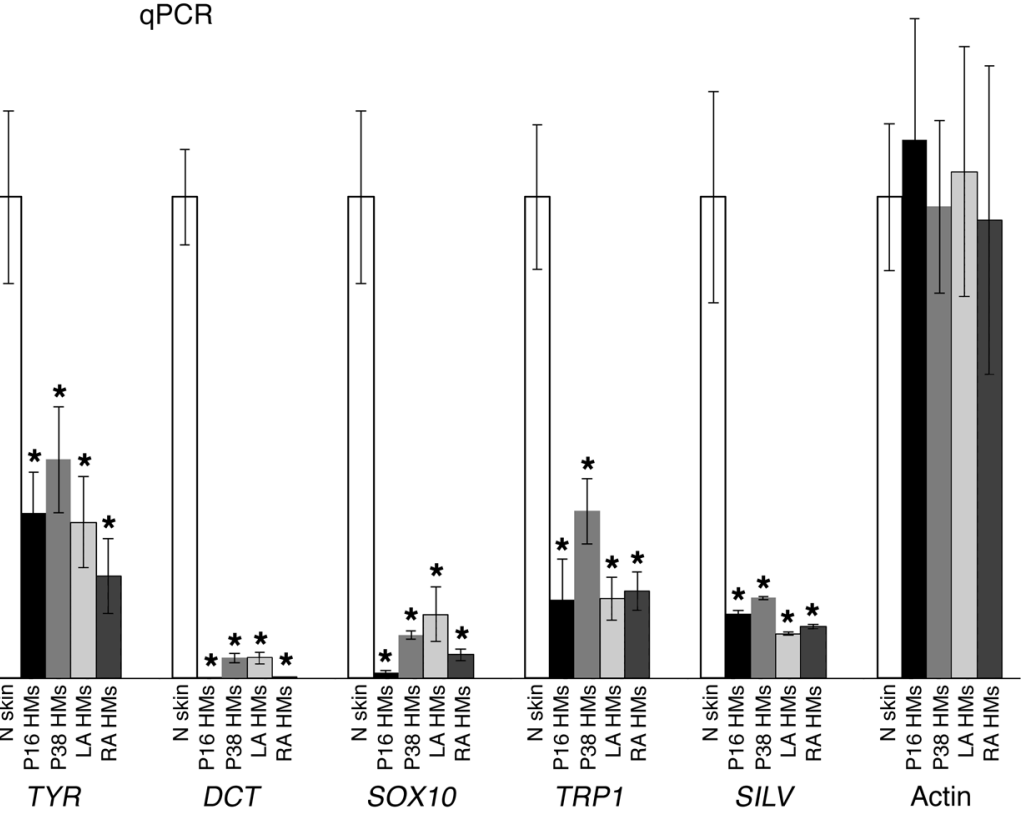

qPCR
SOX10
TRP1

SILV

Actin

Figure 8. Melanocytes isolated from TSC patients' hypomelanotic macules accumulate early-stage melanosomes. (A) Late-stage melanosomes were prominent in melanocytes from normal skin and much less abundant in melanocytes from hypomelanotic macules (original magnification, $\times 3,000$ ). The bottom panel demonstrates the classic stages (I-IV) of melanosome development (original magnification, $\times 12,000$ ). Note that the P38 hypomelanotic macule was compared with normal skin from P16 and P30. (B) Quantification of relative amounts of stage I-IV melanosomes in control skin (N) and hypomelanotic macule melanocytes (HMs). Data represent the mean \pm SD $(n=10)$. ${ }^{*} P<0.05$, by Student's $t$ test versus normal skin control. (C) qPCR analysis of mRNA levels of TSC1, TSC2, and genes involved in pigment formation including MITF, TYR, DCT, SOX10, TRP1, and SILV in melanocytes isolated from patients with TSC, as indicated in both normal skin ( $\mathrm{N}$ skin) and hypomelanotic macules. Note the marked difference in expression of TSC2 and pigment genes. GAPDH served as a control. ${ }^{*} P<0.05$, by Student's $t$ test versus normal skin control. P16 and P38 are TSC subjects with single HM biopsies and cultures. One patient had two biopsies, LA: left leg, RA: right leg.

changes on the intact WT allele of TSC2 (not the one bearing the germline mutation) had occurred and explain the lack of TSC2 expression by these cultures. Random monoallelic expression has been identified in a substantial proportion of human genes in different developmental contexts (78) and may occur in human melanocytes for TSC2. However, further investigations to prove this model will be required.
Previous studies have explored the interaction between TSC1/ TSC2, the Wnt signaling pathway, and GSK3 $\beta$. $\beta$-Catenin levels have been reported to be high in kidney cystadenomas occurring in $\mathrm{Tsc2}^{+/-}$rat and mouse models $(79,80)$ as well as in human kidney angiomyolipomas and pulmonary lymphangioleiomyomatosis (LAM) $(80,81)$. All of these tumors have been shown to have 2-hit inactivation of Tsc2/TSC2 or Tsc1/TSC1. In vitro studies have 
A

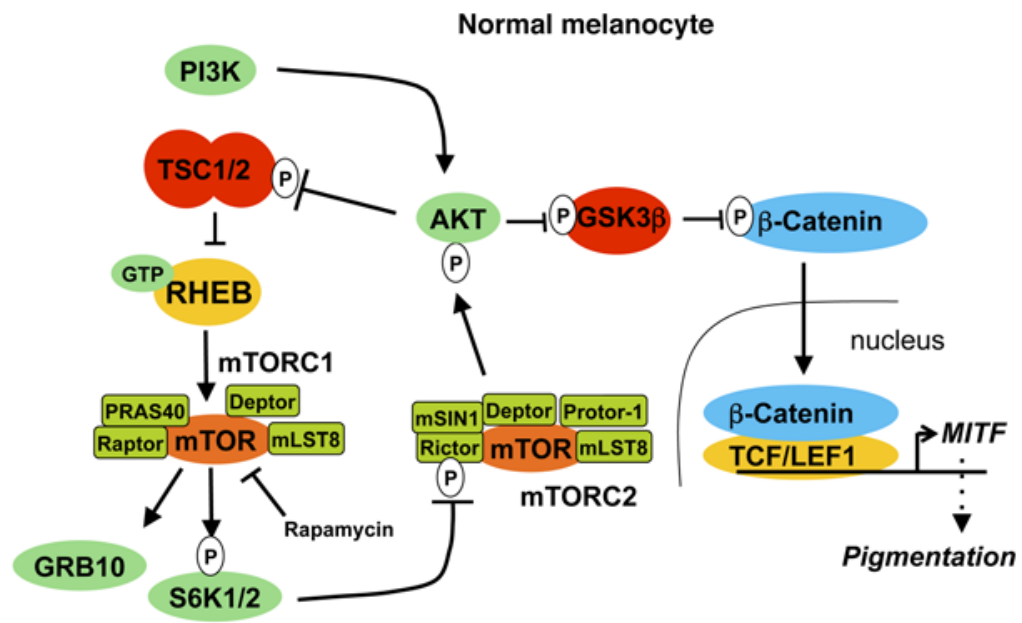

B

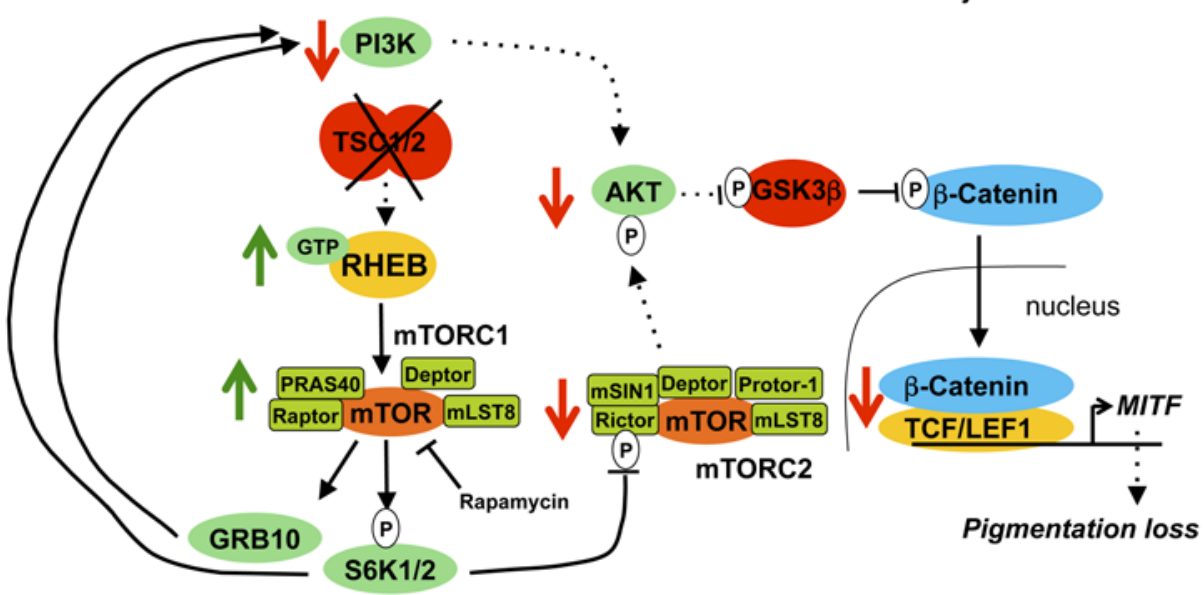

Figure 9. Model of interactions in mTOR signaling in normal and TSC melanocytes. (A) In normal melanocytes, there is physiological activation of PI3K and AKT and inactivation of the TSC complex (TSC1/ TSC2), leading to mTORC1 activity, but retained AKT activity, which then leads to phosphorylation of CSK3 $\beta$, reduced $\beta$-catenin phosphorylation, nuclear entry of $\beta$-catenin, activation of TCF/LEF1 enhancer activity, expression of MITF, and expression of MITF target genes, all of which lead to pigment formation. (B) In TSC hypomelanotic macule melanocytes, there is complete loss of either TSC 1 or TSC2, leading to high levels of RHEB-CTP, mTORC 1 activation, S6K $1 / 2$ activation, inactivation of AKT by several mechanisms, activation of GSK3 $\beta$, phosphorylation of $\beta$-catenin, and reduced levels of nuclear $\beta$-catenin, leading to reduced transcription of MITF and reduced pigmentation. For both $\mathbf{A}$ and $\mathbf{B}$, arrows indicate activating interactions, while solid lines indicate inhibitory interactions. Dotted lines indicate interactions attenuated in hypomelanotic macule melanocytes; green arrows indicate proteins with enhanced activity, and red arrows indicates those with reduced activity. $P$, phosphorylation; PRAS40, AKT1 substrate 1; mLST8, mTOR-associated protein, LST8 homolog; mSIN1, mammalian stress-activated protein kinase-interacting protein. shown that the TSC protein complex binds to multiple components of the Wnt signaling pathway, including GSK3 $\beta$, AXIN, and DSH, with regulation by Wnt stimulation $(79,80)$. However, more recent studies have shown that the core TSC complex consists of TSC1, TSC2, and TBC1D7, without Wnt signaling pathway components (4), suggesting that these previous associations were either cell-type specific or transient in nature. Our results demonstrate that in melanocytes and melanoma cells, loss of the TSC protein complex leads to the opposite effect of markedly reducing $\beta$-catenin levels and, consequently, to loss of MITF and pigment gene expression. We suspect that the effects of TSC1/TSC2 loss on GSK3 $\beta$ activation depend on cell type and developmental context.

GSK3 $\beta$ is known to reside in different pools within the cell, including the $\beta$-catenin destruction complex that is part of the Wnt signaling pathway, as well as a free pool that is accessible to phosphorylation and regulation by AKT and other AGC (protein kinases A, G, and C) family of protein kinases (48). The degree of interchange among these pools is debated, with some studies showing strict segregation of the effects of signaling events on GSK3 $\beta$ in the $\beta$-catenin destruction complex versus the GSK3 $\beta$ present in the free pool $(82,83)$, and others showing some degree of exchange and crosstalk between signaling events affecting these 2 pools $(84,85)$. In addition, although AKT is the canonical kinase regulating GSK3 $\beta$ through phosphorylation, in cells with constitutive
mTORC1 activation due to loss of the TSC protein complex, S6K may phosphorylate GSK3 $\beta$ at the same Ser9 site (86).

It is important to note that all studies examining pigmentation in this report were performed under chronic growth conditions, a minimum of 4 days, allowing development of long-term effects from knockdown or enhanced protein expression and/or drug exposure (e.g., rapamycin, CHIR-99021). Thus, under these chronic conditions, it is likely that there is an exchange of GSK3 $\beta$ within different cell compartments, contributing to the inhibition of $\beta$-catenin signaling downstream of AKT inactivation, as we have shown. Nonetheless, apart from the obvious cell lineage differences, it remains unclear why $\beta$-catenin expression is high in TSC-related kidney angiomyolipomas and pulmonary LAM and low in melanocytes from hypomelanotic macules, in which the same genetic lesion (loss of TSC1 or TSC2) has been sustained.

Some clues to this discrepancy may relate to the biology of MITF in melanocytes and its relationship to Wnt/ $\beta$-catenin. In contrast to neural tissues, in which Wnt $/ \beta$-catenin signaling is associated with proliferation and reduced differentiation, in the melanocyte lineage, this pathway supports both proliferation and differentiation through MITF (42). Indeed, the lineage commitment of early melanocytes from the neural crest requires Wntmediated $\beta$-catenin coactivation of MITF expression (60). However, the requirement for this signaling cascade during develop- 
ment appears to be transient (87). Nonetheless, MITF expression remains important for pigment biosynthesis as well as survival of melanocytes after development, although MITF levels are modulated by the differentiation state and bioenergetic needs of the cell $(53,88)$. Combined with our observations, we speculate that heightened signaling through mTORC1/GSK3 $\beta$ may support a less differentiated phenotype in melanocytes with reduced MITF-regulated pigment biosynthetic pathway activity, while mTORC2/ AKT signaling may promote increased differentiation and pigment biosynthesis. This dual regulatory mechanism, with 1 branch of mTOR signaling (mTORC1) reducing pigment synthesis and the other (mTORC2) promoting it, has not, to our knowledge, been appreciated previously. An earlier study did report that high MITF expression enhances mTORC1 activity, suggesting that there may be a reciprocal regulatory relationship, such that high MITF levels promote mTORC1 activity, while mTORC1 inhibits MITF activity (89). However, these latter effects are mainly seen in cell types other than melanocytes, since melanocyte-specific MITF does not have the same Rag-binding sites as other MITF isoforms or the transcription factors TFEB or TFE3 (89).

In aggregate, these findings indicate that the TSC/mTORC1/ AKT/GSK3 $\beta / \beta$-catenin/MITF axis plays a central role in regulating melanogenesis in humans. Thus, interventions that enhance or diminish mTORC1 activity in melanocytes have a potentially broad role in the modification of pigmentation. Furthermore mTORC1 inhibitors may have a role in the treatment of the subset of malignant melanoma in which growth is dependent on MITF expression.

\section{Methods}

Subjects. Five of six subjects were patients with TSC who were seen at the NHLBI's NIH Clinical Center. One patient with TSC provided samples from an off-site location. A diagnosis of TSC was based on the presence of at least 2 major or 1 major and 2 or more minor diagnostic features (2).

Cell culture. The human melanoma cell line SK-MEL-30 and HEK293T cells were obtained from the Broad Institute of Harvard and MIT (Cambridge, Massachusetts, USA) and the American Type Culture Collection (ATCC), respectively, and were cultured in high-glucose DMEM supplemented with $10 \%$ FBS and incubated at $37^{\circ} \mathrm{C}$ in $5 \% \mathrm{CO}_{2}$. iHPMs transduced with pBABE-hygro-hTERT, pLNCX2CDK4(R24C), and pBABE-puro-p53DD were generated as described previously (51) and cultured in Medium 254 (Invitrogen, Thermo Fisher Scientific). Skin biopsies of hypomelanotic macules and nearby normal skin were obtained for cell culture. Fibroblasts were isolated as described previously (21). The melanocytes were isolated as described previously (90). Briefly, skin samples were cut into small pieces and digested with $0.25 \%$ trypsin overnight at $4^{\circ} \mathrm{C}$. The next day, the epidermal fragment was separated from the dermis with forceps and incubated with $0.02 \%$ EDTA for 10 minutes at $37^{\circ} \mathrm{C}$ and then vortexed to yield a single-cell suspension. The single-cell suspension was then plated in Medium 254 and maintained at $37^{\circ} \mathrm{C}$ in $5 \% \mathrm{CO}_{2}$. Medium was changed 3 times per week.

Transfection and lentiviral and retroviral infections. To generate stable knockdown of TSC1 or TSC2 in SK-MEL-30 cells and iHPMs, human-specific shRNAs and scrambled shRNA controls in PLKO1. puro against TSC1 or TSC2 were cotransfected with psPAX2 (plasmid 12260; Addgene) and pMD2.G (plasmid 12259; Addgene) in 293-T (ATCC) using Lipofectamine 2000 (Invitrogen, Thermo Fisher Sci- entific). Lentiviruses were collected after 48 hours, filtered through a $0.45-\mu \mathrm{m}$ syringe cellulose filter, and cells were then infected for 24 hours in the presence of polybrene $(8 \mu \mathrm{g} / \mathrm{ml})$ and subsequently selected with puromycin $(2 \mu \mathrm{g} / \mathrm{ml})$ for 3 days.

To generate cells with stable expression of WT TSC2, P429S-mutant TSC2, MITF, WT $\beta$-catenin, and the CA form of $\beta$-catenin (S33Y), $293 \mathrm{~T}$ cells were cotransfected with the corresponding cDNA constructs in pBabe, VSV-G, and pUMVC (plasmid 8449; Addgene) plasmids using Lipofectamine 2000. Retroviruses were harvested after 48 hours and sterile filtered. SK-MEL-30 cells and iHPMs expressing shTSC1 or shTSC2 were infected with retroviruses in the presence of polybrene $(8 \mu \mathrm{g} / \mathrm{ml})$. After 24 hours, cells were selected with puromycin $(2 \mu \mathrm{g} / \mathrm{ml})$ or hygromycin $(250 \mu \mathrm{g} / \mathrm{ml})$ for 3 days.

Plasmids. Retroviral IRES-hygro constructs expressing human TSC2 or a patient-derived TSC2 missense were described previously (86). Retroviral pBMN-2 $\beta$-catenin WT and a CA form of $\beta$-catenin (S33Y) constructs were gifts of Ken Inoki (University of Michigan, Ann Arbor, Michigan, USA) (85). pLKO1 shRNA constructs against TSC1, TSC2, and GSK $3 \beta$ were purchased from GE Healthcare with the following sequences: shTSC1-1 (RHS3979-201793839), 5'-CCGGAAAAGGATAACCCAGGTGTTTCTCGAGAAACACCTGGGTTATCCTTTTTTTTTG-3'; shTSC1-2 (RHS3979-201768589), 5'-CCGGGCAGCCATCTTGGAAGCATAACTCGAGTTATGCTTCCAAGATGGCTGCTTTTTG-3'; shTSC1-3 (RHS3979-201792689), 5'-CCGGAAAGAAGAAGCTGCAATATCTCTCGAGAGATATTGCAGCTTCTTCTTTTTTTTG-3'; shTSC2-1 (RHS3979-200795892), 5'-AAACCCAGGGCTGCCTTGGAAAAG-3'; shTSC2-2: TRCNO000040179 (RHS3979-200795893), 5'-AAACCCAGGGCTGCCTTGGAAAAG-3'; shTSC2-3 (RHS3979200795895), 5'-AAACCCAGGGCTGCCTTGGAAAAG-3'; shGSK3ß-1 (RHS3979-201768854), 5'-AAACCCAGGGCTGCCTTGGAAAAG-3'; shGSK3ß-2 (RHS3979-201768852), 5'-AAACCCAGGGCTGCCTTGGAAAAG-3'; and shGSK3ß-3 (RHS3979-201742129), 5'-AAACCCAGGGCTGCCTTGGAAAAG-3'. shRaptor and shRictor constructs were gifts of David Sabatini (MIT, Boston, Massachusetts, USA). Plasmids 1858 and 1853 were obtained from Addgene.

Real-time quantitative RT-PCR. Total RNA was isolated using TRIzol reagent (catalog 15596026; Invitrogen, Thermo Fisher Scientific) following the manufacturer's instructions. cDNA was synthesized using 500 ng total RNA and the cDNA Synthesis Kit with SuperScript III RT (catalog 11752050; Life Technologies, Thermo Fisher Scientific) according to the manufacturer's instructions. cDNA was diluted 1:100 before quantification by real-time quantitative PCR (qPCR). The PCR reactions contained $10 \mu \mathrm{l} 2 \times$ iQ SYBR Green Supermix (cata$\log 170-882$; Bio-Rad), $0.25 \mu \mathrm{M}$ of each primer, and $3 \mu$ diluted cDNA. Reactions were processed for 1 cycle at $94^{\circ} \mathrm{C}$ for 3 minutes, then for 45 cycles at $94^{\circ} \mathrm{C}$ for 10 seconds, and for 40 seconds at $68^{\circ} \mathrm{C}$ using the Applied Biosystems Step One Plus instrument. The primers used in this study, including MITF-M, TYR, TYRP1, DCT, SOX10, and SILV (53) and TSC1 (91) were described previously. The primers used for TSC2 expression were as follows: forward, $5^{\prime}$-GAAGTCGCAAAAACCAAGAA-3'; reverse, 5'-TGTGTCTCCCATTGTCTGTG-3'. Relative expression was measured using the $2^{\triangle \mathrm{CT}}$ method, and data are represented as fold levels over control.

Immunoblot analysis. Preparation of protein extracts and immunoblot analysis were performed as described previously (92). The following antibodies used in Western blotting were purchased from Cell Signaling Technology: Flag (product 2368); TSC1 (product 4906); TSC2 
(product 3612); S6 (product 2217); p-S6 (Ser235/236; product 4858); p-S6 (Ser240/244; product 2215); p70 S6K (product 2708); p-p70 S6K (Thr389; product 9205); 4E-BP1 (product 9452); p-4E-BP1 (Thr37/46; product 2855); GSK3 $\beta$ (product 9316); p-GSK3 $\beta$ (Ser9; product 9336); $\beta$-catenin (product 9562); $\mathrm{p}-\beta$-catenin (Ser33/37, Thr41); AKT (pan) (product 40D4); and p-AKT (Ser473; product 736E11; Thr308; product 4056). p-p70 S6K (Thr229) was purchased from Signalway Antibody LLC (product 12021); TYR was purchased from Abcam (cata$\log$ ab61294); and PGC-1 $\alpha$ (catalog sc-5816) and Topo II $\beta$ (catalog sc-13059) were purchased from Santa Cruz Biotechnology Inc. $\beta$-Tubulin (catalog T8328) and $\beta$-actin (catalog A2228) were purchased from Sigma-Aldrich. MITF antibody was purchased from Thermo Fisher Scientific (catalog MA5-14146).

Subcellular fractionations. Cytoplasmic and nuclear fractionations were isolated as described previously (92). $\beta$-Tubulin and Topo II $\beta$ were used as controls for cytoplasmic and nuclear fractions, respectively.

IHC. Immunohistochemical studies were performed on paraffin-embedded skin biopsies from TSC patients' hypomelanotic macules and adjacent normal-appearing skin. Primary antibodies used included anti-MITF (1:50; M362129-2; Dako); anti-p-S6 (Ser240/244) (1:100; 2215; Cell Signaling Technology); and anti-MelanA (1:200; ab731; Abcam). MITF and p-S6 were detected using the Dako DAB Detection Kit, and MelanA was detected with the AEC Detection Kit (Dako EnVision+ System HRP). Costaining for p-S6 (Ser240/244) and MelanA was performed. All immunohistochemical studies were performed at the histopathology core of Brigham and Women's Hospital.

Luciferase reporter assays. For luciferase reporter assays, SK-MEL30 cells or iHPMs with shScrambled (shScr) or shTSC1 or shTSC2 were seeded in a 12-well plate in triplicate and transfected the following day using Lipofectamine 2000 (Invitrogen, Thermo Fisher Scientific). Each transfection contained $250 \mathrm{ng}$ of the firefly luciferase reporter plasmid pGL3-MITF-M or TK-FOP/TK-TOP and $12.5 \mathrm{ng}$ pRL-CMV Renilla luciferase control, and the total DNA was adjusted to $2 \mu \mathrm{g}$ with pcDNA3. Where indicated, rapamycin $(20 \mathrm{nM}$; Figure 2, A-H; Figure 3, B and D, F-H; Supplemental Figure 2, A-H; Supplemental Figure 3, B and D, F-H), AR-A014418 (5 $\mu \mathrm{M}$; Figure 5, B, E and G; Figure 6, C and D; Supplemental Figure 5, F-J), CHIR99021 ( $3 \mu \mathrm{M}$; Figure 5, A, D and F; Figure 6, A and B; Supplemental Figure $5, \mathrm{~A}-\mathrm{E})$, or BIO ( $3 \mu \mathrm{M}$; Figure $5, \mathrm{C}$ and $\mathrm{H}$; Figure $6, \mathrm{E}$ and $\mathrm{F}$; Supplemental Figure 5, K-N) was added for 72 hours prior to measuring luciferase levels. The next day, cells were lysed in $200 \mu \mathrm{l}$ of $1 \times$ passive lysis buffer (Promega), and luciferase levels were measured using a Dual-Glo Luciferase Kit (catalog E2920; Promega) on a GloMax 20/20 luminometer (Promega). Luciferase values were normalized to constitutive Renilla luciferase, and for the TK-FOP/ TK-TOP experiments, values were normalized to pRL-TK (Promega) as described previously (42).

DNA isolation and next-generation sequencing. Genomic DNA was isolated from the fibroblasts and melanocytes cultured from the hypomelanotic macules and paired normal skin from TSC patients using a DNeasy Blood and Tissue Kit (catalog 69504; QIAGEN). Next-generation sequencing of the DNA samples from cultured fibroblasts and melanocytes for the mutational status of TSC1 and TSC2 was performed as described previously (21) (dbGaP phs001236.v1).

DNA methylation analysis. Genomic DNA extracted from primary melanocytes from hypomelanotic macules and paired normal skin was subjected to bisulfite treatment using the EpiTect Bisul- fite Kit (catalog 59104; QIAGEN) according to the manufacturer's instructions. The primers used for amplification of regions of the CpG island upstream of the human TSC2 locus were as follows: DMTSC2F， 5'-TTYGTTAGAGGGYGGTATAGAAT-3'; DMTSC2R, 5'-ACACTACRAAATCCRCCTCTC-3'; 1272F, TTTGTTTTATATGGGTAGAGGAGAGA; 1484R, ACTCCCTAAAAAAACTCAACTACAAC; 391F, GGATAGTAAGTTTATTGTAGGAGGG; and 683R, AAATAAAAACTACAAACTAAAATCCC (corresponding chromosome [chr] 16:2047897-2048119, chr16: 2048260-2048472, and chr16: 2047379-2047646). PCR products were subjected to Sanger sequencing and compared with a control, universal methylated human DNA standard (catalog D5011; Zymo Research).

Melanin content measurement. Melanin content was measured as described previously (93). Briefly, the cells were harvested, pelleted, and washed in PBS. Half of the cells were used for protein amount determination. The other half was resuspended in $400 \mu \mathrm{l}$ of $1 \mathrm{~N} \mathrm{NaOH}$ solution and incubated at room temperature for 2 hours, and $100 \mu \mathrm{l}$ of the solution was then loaded onto a 96-well plate. The melanin content was determined by measuring the absorbance of the solution between 405 and $490 \mathrm{~nm}$ and compared with a melanin standard (product M8631; Sigma-Aldrich). Melanin content was expressed as micrograms per milligram of protein.

TYR activity assays. TYR activity was determined as described previously (94). Briefly, cells were sonicated in protein lysis buffer. After protein quantification, $250 \mu \mathrm{g}$ total protein lysate in a total volume of $100 \mu$ lysis buffer was loaded onto a $96-$ well plate, followed by the addition of $100 \mu \mathrm{l}$ of $1 \mathrm{mM}$ L-DOPA (product D9628; Sigma-Aldrich). The plate was incubated at $37^{\circ} \mathrm{C}$ for 1 hour, and the absorbance at $475 \mathrm{~nm}$ was recorded. TYR activity was expressed as a percentage of the control.

TEM. Nonimmortalized primary melanocytes at passage 2 isolated from hypomelanotic macule biopsies and paired nearby normal skin from patients with TSC were fixed in $2 \%$ glutaraldehyde $/ 2 \%$ formaldehyde in cacodylate buffer, followed by $1 \%$ osmium tetroxide. The samples were embedded in epoxy resin, cut into 60- to 80-nm sections, and viewed with an FEI Tecnai $12 \mathrm{TEM}$ operated at $80 \mathrm{kV}$ and $\times 9,300$ magnification (Harvard Medical School Electron Microscopy core facility). The number of stage I-IV melanosomes were quantified in a minimum of 10 cells from each melanocyte culture and used to calculate the percentages of stage I-IV melanosomes as described previously (43).

Statistics. All data are presented as the mean \pm SD from at least 3 independent experiments. A 2-tailed Student's $t$ test was used to compare group differences in all cases, and a $P$ value of less than 0.05 was considered statistically significant.

Study approval. This study was approved by the IRB of the NHLBI (protocols 96- $\mathrm{H}-0100$ and 00-H-0051). All subjects providing biopsy samples were aged 18 years or older and provided written informed consent.

\section{Author contributions}

JC designed the research studies, conducted experiments, acquired data, analyzed data, and wrote the manuscript. MET conducted experiments, acquired data, analyzed data, and reviewed the manuscript. JM provided samples from the TSC patients seen at the NIH and reviewed the manuscript. TND acquired biopsies from the patients seen at the NIH and reviewed the data and manuscript. HRW helped design the studies, contributed many reagents, acquired, analyzed, and reviewed the data, and helped 
write the manuscript. DJK designed the research studies, analyzed data, and wrote the manuscript.

\section{Acknowledgments}

We thank Brendan Manning's laboratory (T. H. Chan School of Public Health, Harvard University, Boston, Massachusetts, USA) for providing TSC2-knockdown cells, cDNA constructs, and GSK3 $\beta$ inhibitors and Ken Inoki for providing $\beta$-catenin constructs. We also thank the members of DJK's laboratory for helpful comments and suggestions. This work was supported by grants from the National Cancer Institute (NCI), NIH (1P01CA120964, to DJK and R01AR062080, to TND). JM was supported by the Intramural Research Program of the NHLBI, NIH.

Address correspondence to: David J. Kwiatkowski, Brigham and Women's Hospital, 20 Shattuck Street Thorn 826, Boston, Massachusetts 02115, USA. Phone: 857.307.0781; E-mail: dk@rics. bwh.harvard.edu.
1. Kwiatkowski DJ, Thiele EA, Whittemore VH, eds. Tuberous Sclerosis Complex: Genes, Clinical Features and Therapeutics. Weinheim, Germany: Wiley-VCH; 2010.

2. Northrup H, Krueger DA, International Tuberous Sclerosis Complex Consensus Group. Tuberous sclerosis complex diagnostic criteria update: recommendations of the 2012 International Tuberous Sclerosis Complex Consensus Conference. Pediatr Neurol. 2013;49(4):243-254.

3. Kwiatkowski DJ. Genetics of tuberous sclerosis complex In: Kwiatkowski DJ, Whittemore VH, Thiele EA eds. Tuberous Sclerosis Complex: Genes, Clinical Features and Therapeutics. Weinheim, Germany: Wiley-VCH; 2010:29-60.

4. Dibble CC, et al. TBC1D7 is a third subunit of the TSC1-TSC2 complex upstream of mTORC1. Mol Cell. 2012;47(4):535-546.

5. Garami A, et al. Insulin activation of Rheb, a mediator of $\mathrm{mTOR} / \mathrm{S} 6 \mathrm{~K} / 4 \mathrm{E}-\mathrm{BP}$ signaling, is inhibited by TSC1 and 2. Mol Cell. 2003;11(6):1457-1466.

6. Inoki K, Li Y, Xu T, Guan KL. Rheb GTPase is a direct target of TSC2 GAP activity and regulates mTOR signaling. Genes Dev. 2003;17(15):1829-1834.

7. Saucedo LJ, Gao X, Chiarelli DA, Li L, Pan D, Edgar BA. Rheb promotes cell growth as a component of the insulin/TOR signalling network. Nat Cell Biol. 2003;5(6):566-571.

8. Stocker $\mathrm{H}$, et al. Rheb is an essential regulator of S6K in controlling cell growth in Drosophila. Nat Cell Biol. 2003;5(6):559-565.

9. Tee AR, Manning BD, Roux PP, Cantley LC, Blenis J. Tuberous sclerosis complex gene products, Tuberin and Hamartin, control mTOR signaling by acting as a GTPase-activating protein complex toward Rheb. Curr Biol. 2003;13(15):1259-1268.

10. Zhang $\mathrm{H}$, et al. Loss of Tsc1/Tsc 2 activates mTOR and disrupts PI3K-Akt signaling through downregulation of PDGFR. J Clin Invest. 2003;112(8):1223-1233.

11. Zhang Y, et al. Coordinated regulation of protein synthesis and degradation by mTORC1. Nature. 2014;513(7518):440-443.

12. El-Hashemite N, Zhang H, Henske EP, Kwiatkowski DJ. Mutation in TSC2 and activation of mammalian target of rapamycin signalling pathway in renal angiomyolipoma. Lancet. 2003;361(9366):1348-1349.

13. Harrington LS, et al. The TSC1-2 tumor suppressor controls insulin-PI3K signaling via regulation of IRS proteins. J Cell Biol. 2004;166(2):213-223.

14. Shah OJ, Hunter T. Turnover of the active fraction of IRS1 involves raptor-mTOR- and
S6K1-dependent serine phosphorylation in cell culture models of tuberous sclerosis. Mol Cell Biol. 2006;26(17):6425-6434.

15 . Um SH, et al. Absence of S6K1 protects against ageand diet-induced obesity while enhancing insulin sensitivity. Nature. 2004;431(7005):200-205.

16. Zhang $\mathrm{H}$, et al. PDGFRs are critical for PI3K/Akt activation and negatively regulated by mTOR. JClin Invest. 2007;117(3):730-738.

17. Huang J, Dibble CC, Matsuzaki M, Manning BD. The TSC1-TSC2 complex is required for proper activation of mTOR complex 2. Mol Cell Biol. 2008;28(12):4104-4115.

18. Yu Y, et al. Phosphoproteomic analysis identifies Grb10 as an mTORC1 substrate that negatively regulates insulin signaling. Science. 2011;332(6035):1322-1326.

19. Hsu PP, et al. The mTOR-regulated phosphoproteome reveals a mechanism of mTORC1-mediated inhibition of growth factor signaling. Science. 2011;332(6035):1317-1322.

20. Darling T, Mauser M, Moss J. Dermatologic Manifestations of Tuberous Sclerosis Complex (TSC). In: Kwiatkowski DJ, Thiele E, Whittemore V eds. T Tuberous Sclerosis Complex: Genes, Clinical Features and Therapeutics. Weinheim, Germany: Wiley-VCH; 2010:285-310.

21. Tyburczy ME, et al. Sun exposure causes somatic second-hit mutations and angiofibroma development in tuberous sclerosis complex. Hum Mol Genet. 2014;23(8):2023-2029.

22. Jimbow K, Fitzpatrick TB, Szabo G, Hori Y. Congenital circumscribed hypomelanosis: a characterization based on electron microscopic study of tuberous sclerosis, nevus depigmentosus, and piebaldism. J Invest Dermatol. 1975;64(1):50-62.

23. Jóźwiak S, Schwartz RA, Janniger CK, Michałowicz R, Chmielik J. Skin lesions in children with tuberous sclerosis complex: their prevalence, natural course, and diagnostic significance. Int J Dermatol.1998;37(12):911-917.

24. Gold AP, Freeman JM. Depigmented nevi: the earliest sign of tuberous sclerosis. Pediatrics. 1965;35:1003-1005.

25. Wataya-Kaneda M, et al. Clinical and histologic analysis of the efficacy of topical rapamycin therapy against hypomelanotic macules in tuberous sclerosis complex. JAMA Dermatol. 2015;151(7):722-730.

26. Hong L, Simon JD, Sarna T. Melanin structure and the potential functions of uveal melanosomes. Pigment Cell Res. 2006;19(5):465-466.

27. Sarna T. Properties and function of the ocular melanin--a photobiophysical view. JPhotochem Photobiol B, Biol.1992;12(3):215-258.
28. Wang Z, Dillon J, Gaillard ER. Antioxidant properties of melanin in retinal pigment epithelial cells. Photochem Photobiol. 2006;82(2):474-479.

29. Weis E, Shah CP, Lajous M, Shields JA, Shields CL. The association between host susceptibility factors and uveal melanoma: a meta-analysis. Arch Ophthalmol. 2006;124(1):54-60.

30. Weiter JJ, Delori FC, Wing GL, Fitch KA. Relationship of senile macular degeneration to ocular pigmentation. Am JOphthalmol. 1985;99(2):185-187.

31. Hu DN, Savage HE, Roberts JE. Uveal melanocytes, ocular pigment epithelium, and Müller cells in culture: in vitro toxicology. Int J Toxicol. 2002;21(6):465-472.

32. Oetting WS, King RA. Molecular basis of albinism: mutations and polymorphisms of pigmentation genes associated with albinism. Hum Mutat. 1999;13(2):99-115.

33. Shoag J, et al. PGC-1 coactivators regulate MITF and the tanning response. Mol Cell. 2013;49(1):145-157.

34. Hodgkinson CA, et al. Mutations at the mouse microphthalmia locus are associated with defects in a gene encoding a novel basic-helix-loophelix-zipper protein. Cell. 1993;74(2):395-404.

35. Hughes AE, Newton VE, Liu XZ, Read AP. A gene for Waardenburg syndrome type 2 maps close to the human homologue of the microphthalmia gene at chromosome 3p12-p14.1. Nat Genet. 1994;7(4):509-512.

36. Tassabehji M, Newton VE, Read AP. Waardenburg syndrome type 2 caused by mutations in the human microphthalmia (MITF) gene. Nat Genet. 1994;8(3):251-255.

37. Goding CR. Mitf from neural crest to melanoma: signal transduction and transcription in the melanocyte lineage. Genes Dev. 2000;14(14):1712-1728.

38. Steingrímsson E, Copeland NG, Jenkins NA. Melanocytes and the microphthalmia transcription factor network. Annu Rev Genet. 2004;38:365-411.

39. Wodarz A, Nusse R. Mechanisms of Wnt signaling in development. Annu Rev Cell Dev Biol. 1998;14:59-88.

40. Cadigan KM, Nusse R. Wnt signaling: a common theme in animal development. Genes Dev. 1997;11(24):3286-3305.

41. Ikeya M, Lee SM, Johnson JE, McMahon AP, Takada S. Wnt signalling required for expansion of neural crest and CNS progenitors. Nature. 1997;389(6654):966-970.

42. Widlund HR, et al. Beta-catenin-induced melanoma growth requires the downstream target Microphthalmia-associated transcription factor. JCell Biol. 2002;158(6):1079-1087. 
43. Ho H, Kapadia R, Al-Tahan S, Ahmad S, Ganesan AK. WIPI1 coordinates melanogenic gene transcription and melanosome formation via TORC1 inhibition. J Biol Chem. 2011;286(14):12509-12523.

44. Behrens J, et al. Functional interaction of beta-catenin with the transcription factor LEF-1. Nature. 1996;382(6592):638-642.

45. Huber O, Korn R, McLaughlin J, Ohsugi M, Herrmann BG, Kemler R. Nuclear localization of beta-catenin by interaction with transcription factor LEF-1. Mech Dev. 1996;59(1):3-10.

46. Molenaar M, et al. XTcf-3 transcription factor mediates beta-catenin-induced axis formation in Xenopus embryos. Cell. 1996;86(3):391-399.

47. Jope RS, Yuskaitis CJ, Beurel E. Glycogen synthase kinase-3 (GSK3): inflammation, diseases, and therapeutics. Neurochem Res. 2007;32(4-5):577-595.

48. Beurel E, Grieco SF, Jope RS. Glycogen synthase kinase-3 (GSK3): regulation, actions, and diseases. Pharmacol Ther. 2015;148:114-131.

49. Wilkie AL, Jordan SA, Jackson IJ. Neural crest progenitors of the melanocyte lineage: coat colour patterns revisited. Development. 2002;129(14):3349-3357.

50. Jeong HS, et al. Involvement of mTOR signaling in sphingosylphosphorylcholine-induced hypopigmentation effects. J Biomed Sci. 2011;18:55.

51. Garraway LA, et al. Integrative genomic analyses identify MITF as a lineage survival oncogene amplified in malignant melanoma. Nature. 2005;436(7047):117-122.

52. Lin JY, Fisher DE. Melanocyte biology and skin pigmentation. Nature. 2007;445(7130):843-850.

53. Haq R, et al. Oncogenic BRAF regulates oxidative metabolism via PGC1 $\alpha$ and MITF. Cancer Cell. 2013;23(3):302-315.

54. Ohguchi K, Banno Y, Nakagawa Y, Akao Y, Nozawa $Y$. Negative regulation of melanogenesis by phospholipase D1 through mTOR/p70 S6 kinase 1 signaling in mouse B16 melanoma cells. JCell Physiol. 2005;205(3):444-451.

55. Buscà R, Bertolotto C, Ortonne JP, Ballotti R. Inhibition of the phosphatidylinositol 3-kinase/ p70(S6)-kinase pathway induces B16 melanoma cell differentiation. J Biol Chem. 1996;271(50):31824-31830.

56. Thoreen CC, et al. An ATP-competitive mammalian target of rapamycin inhibitor reveals rapamycin-resistant functions of mTORC1. J Biol Chem. 2009;284(12):8023-8032.

57. Hajduch E, Litherland GJ, Hundal HS. Protein kinase $\mathrm{B}(\mathrm{PKB} / \mathrm{Akt})--\mathrm{a}$ key regulator of glucose transport? FEBS Lett. 2001;492(3):199-203.

58. Cross DA, Alessi DR, Cohen P, Andjelkovich M, Hemmings BA. Inhibition of glycogen synthase kinase- 3 by insulin mediated by protein kinase $B$. Nature. 1995;378(6559):785-789.

59. MacDonald BT, Tamai K, He X. Wnt/beta-catenin signaling: components, mechanisms, and diseases. Dev Cell. 2009;17(1):9-26.

60. Dorsky RI, Raible DW, Moon RT. Direct regulation of nacre, a zebrafish MITF homolog required for pigment cell formation, by the Wnt pathway. Genes Dev. 2000;14(2):158-162.

61. Takeda K, et al. Induction of melanocyte-specific microphthalmia-associated transcription factor by Wnt-3a. J Biol Chem. 2000;275(19):14013-14016.

62. van de Wetering $\mathrm{M}$, et al. Armadillo coactivates transcription driven by the product of the Drosophila segment polarity gene dTCF. Cell. 1997;88(6):789-799.

63. Davies SP, Reddy H, Caivano M, Cohen P. Specificity and mechanism of action of some commonly used protein kinase inhibitors. Biochem $\mathrm{J}$. 2000;351(Pt 1):95-105.

64. Bain J, et al. The selectivity of protein kinase inhibitors: a further update. Biochem J. 2007;408(3):297-315.

65. Schroeder JH, Bell LS, Janas ML, Turner M. Pharmacological inhibition of glycogen synthase kinase 3 regulates $\mathrm{T}$ cell development in vitro. PLoS One. 2013;8(3):e58501.

66. Bhat R, et al. Structural insights and biological effects of glycogen synthase kinase 3-specific inhibitor AR-A014418. J Biol Chem. 2003;278(46):45937-45945.

67. Ly PT, et al. Inhibition of GSK3ß-mediated BACE1 expression reduces Alzheimer-associated phenotypes. J Clin Invest. 2013;123(1):224-235.

68. Bellei B, Flori E, Izzo E, Maresca V, Picardo M. GSK3beta inhibition promotes melanogenesis in mouse B16 melanoma cells and normal human melanocytes. Cell Signal. 2008;20(10):1750-1761.

69. Crino PB, Nathanson KL, Henske EP. The tuberous sclerosis complex. N Engl JMed. 2006;355(13):1345-1356.

70. Bissler JJ, et al. Sirolimus for angiomyolipoma in tuberous sclerosis complex or lymphangioleiomyomatosis. N Engl JMed. 2008;358(2):140-151.

71. Bissler JJ, et al. Everolimus for angiomyolipoma associated with tuberous sclerosis complex or sporadic lymphangioleiomyomatosis (EXIST-2): a multicentre, randomised, double-blind, placebocontrolled trial. Lancet. 2013;381(9869):817-824.

72. McCormack FX, et al. Efficacy and safety of sirolimus in lymphangioleiomyomatosis. $\mathrm{N} \mathrm{EnglJ}$ Med. 2011;364(17):1595-1606.

73. Franz DN, et al. Efficacy and safety of everolimus for subependymal giant cell astrocytomas associated with tuberous sclerosis complex (EXIST-1): a multicentre, randomised, placebo-controlled phase 3 trial. Lancet. 2013;381(9861):125-132.

74. Franz DN, et al. Rapamycin causes regression of astrocytomas in tuberous sclerosis complex. Ann Neurol. 2006;59(3):490-498.

75. Fitzpatrick TB. History and significance of white macules, earliest visible sign of tuberous sclerosis. Ann N Y Acad Sci. 1991;615:26-35.

76. De Schepper S, Maertens O, Callens T, Naeyaert JM, Lambert J, Messiaen L. Somatic mutation analysis in NF1 café au lait spots reveals two NF1 hits in the melanocytes. Jinvest Dermatol. 2008;128(4):1050-1053.

77. Hodis $\mathrm{E}$, et al. A landscape of driver mutations in melanoma. Cell. 2012;150(2):251-263.
78. Eckersley-Maslin MA, Spector DL. Random monoallelic expression: regulating gene expression one allele at a time. Trends Genet. 2014;30(6):237-244.

79. Mak BC, Takemaru K, Kenerson HL, Moon RT, Yeung RS. The tuberin-hamartin complex negatively regulates beta-catenin signaling activity. JBiol Chem. 2003;278(8):5947-5951.

80. Mak BC, Kenerson HL, Aicher LD, Barnes EA, Yeung RS. Aberrant beta-catenin signaling in tuberous sclerosis. Am J Pathol. 2005;167(1):107-116.

81. Flavin RJ, Cook J, Fiorentino M, Bailey D, Brown M, Loda MF. $\beta$-Catenin is a useful adjunct immunohistochemical marker for the diagnosis of pulmonary lymphangioleiomyomatosis. Am J Clin Pathol. 2011;135(5):776-782.

82. Ding VW, Chen RH, McCormick F. Differential regulation of glycogen synthase kinase 3 beta by insulin and Wnt signaling. J Biol Chem. 2000;275(42):32475-32481.

83. Ng SS, et al. Phosphatidylinositol 3-kinase signaling does not activate the wnt cascade. J Biol Chem. 2009;284(51):35308-35313.

84. Fukumoto S, et al. Akt participation in the Wnt signaling pathway through Dishevelled.J Biol Chem. 2001;276(20):17479-17483.

85. Inoki K, et al. TSC2 integrates Wnt and energy signals via a coordinated phosphorylation by AMPK and GSK3 to regulate cell growth. Cell. 2006;126(5):955-968.

86. Zhang HH, Lipovsky AI, Dibble CC, Sahin M, Manning BD. S6K1 regulates GSK3 under conditions of mTOR-dependent feedback inhibition of Akt. Mol Cell. 2006;24(2):185-197.

87. Hari L, et al. Temporal control of neural crest lineage generation by Wnt/ $\beta$-catenin signaling. Development. 2012;139(12):2107-2117.

88. Vazquez F, et al. PGC1 $\alpha$ expression defines a subset of human melanoma tumors with increased mitochondrial capacity and resistance to oxidative stress. Cancer Cell. 2013;23(3):287-301.

89. Zhang T, et al. Mitf is a master regulator of the $\mathrm{v}$-ATPase, forming a control module for cellular homeostasis with v-ATPase and TORC1. J Cell Sci. 2015;128(15):2938-2950.

90. Gilchrest BA, Vrabel MA, Flynn E, Szabo G. Selective cultivation of human melanocytes from newborn and adult epidermis. J Invest Dermatol. 1984;83(5):370-376.

91. Khatri S, Yepiskoposyan H, Gallo CA, Tandon P, Plas DR. FOXO3a regulates glycolysis via transcriptional control of tumor suppressor TSC1. J Biol Chem . 2010;285(21):15960-15965.

92. Cao J, et al. MC1R is a potent regulator of PTEN after UV exposure in melanocytes. Mol Cell. 2013;51(4):409-422.

93. Chen KG, et al. Melanosomal sequestration of cytotoxic drugs contributes to the intractability of malignant melanomas. Proc Natl Acad Sci US A. 2006;103(26):9903-9907.

94. Choi TY, et al. Impact of $\mathrm{NAD}(\mathrm{P}) \mathrm{H}$ :quinone oxidoreductase- 1 on pigmentation. J Invest Dermatol. 2010;130(3):784-792. 